\title{
COMMUTATIVITY IN SERIES OF ORDINALS: A STUDY OF INVARIANTS ${ }^{1}$
}

\author{
BY \\ J. L. HICKMAN
}

\begin{abstract}
It is well known that two ordinals are additively commutative if and only if they are finite multiples of some given ordinal, and it is very easy to extend this result to any finite sequence of ordinals. However, no necessary and sufficient conditions for the commutativity of a series of ordinals seem to be known when the length of that series is infinite, although sufficient conditions for certain cases have been given by Sierpinski and Ginsburg. In this paper we present such necessary and sufficient conditions. The general problem is split into five distinct cases: those in which the length of the series is a regular initial ordinal, a singular initial ordinal, an infinite, noninitial prime component, an infinite successor ordinal, and an infinite limit ordinal that is not a prime component. These are dealt with respectively in the second through to the sixth sections of the paper, and it turns out that in every case our criteria can be expressed in terms of an ordinal parameter, which is in fact an invariant of the series in question. This concept of invariance is introduced in the first section, which also contains several lemmas and a slight strengthening of the original Sierpiński-Ginsburg result. The final section of this paper differs from the preceding four sections in two aspects. Firstly, the proofs of its two main results are merely sketched, since they contain no arguments that have not previously appeared in some form or other. Secondly, we have not given any explicit determination of the ordinal parameter introduced in this section, since we felt that such a determination would prolong the paper intolerably and encroach upon work done by J. A. H. Anderson: we have therefore simply referred to Anderson's interesting paper.
\end{abstract}

1. Ordinals are assumed to be defined in such a manner that each is the set of all preceding ordinals: infinite ordinals will generally be denoted by lower-case Greek letters, with " $\omega$ " being reserved for the first such. The lower-case Latin alphabet will be used as follows: the letters " $r$ ", " $s$ ", " $t$ ", ... will denote sequences of ordinals (a sequence is defined to be a function whose domain is an ordinal); the letters " $f$ ", " $g$ ", " $h$ " will denote general functions; and the remaining letters will denote finite ordinals (natural numbers). We include 0 amongst the natural numbers, but in order

Received by the editors October 29, 1976 and, in revised form, December 1, 1977.

AMS (MOS) subject classifications (1970). Primary 04A10.

${ }^{1}$ The work contained in this paper was done while the author was a Research Fellow at the Australian National University. 
to save tiresome repetition we adopt the convention that unless the contrary is either obvious or explicitly stated, any sequence of ordinals referred to in the text consists entirely of nonzero terms.

Given any ordinal $\alpha$, we define an $\alpha$-sequence $s=\left(s_{\xi}\right)_{\xi<\alpha}$ of ordinals to be an ordinal-valued sequence with domain $\alpha$; this domain is denoted by " $o(s)$ ", and is called the "length" of the sequence $s$. It is natural to denote the value of $s$ at an ordinal $\xi<\alpha$ by " $s_{\xi}$ ". The 0 -sequence is denoted by " $\varnothing$ ".

Let two sequences $r, s$ be given. We say that $r$ is a subsequence of $s$ if there is a strictly increasing function $f: o(r) \rightarrow o(s)$ such that $r_{\xi}=s_{f(\xi)}$ for each $\xi<o(r)$. Take any subset $X$ of $o(s)$; then $X$ obviously determines a unique subsequence of $s$, viz. the sequence $r$ such that there is a strictly increasing function $f: o(r) \rightarrow o(s)$ with $f^{\prime \prime} o(r)=X$, and we denote $r$ by " $s[X]$ ". We take this notation one step further by letting $P$ be a predicate (or some other linguistic expression) that defines a unique subset $X$ of $o(s)$, and denoting $s[X]$ by " $s[P]$ ". In particular, we denote by " $s[=\tau]$ ", where $\tau$ is a given ordinal, the subsequence $s\left[s_{\xi}=\tau\right]$, with similar interpretations of $s[\geqslant \tau]$, $s[\geqslant \tau,<\theta]$, and so on. Finally, for any subset $X$ of $o(s)$, we denote $s[o(s)-$ $X]$ by " $s-s[X]$ ", and say that $s[X]$ and $s-s[X]$ are complementary (in $s$ ).

If we are given a subsequence $r$ of a sequence $s$, then in general there will be more than one $X \subseteq o(s)$ for which $r=s[X]$. For the majority of our purposes however, it will not matter which such $X$ is chosen, and so we can simply choose one at random and define $s-r$ accordingly. In those cases where this looseness could result in error, we shall clarify the situation.

Let $r, s$ be two sequences. We call $r$ an "arrangement" of $s$ if there is a bijection $f: o(r) \rightarrow o(s)$ such that $r_{\xi}=s_{f(\xi)}$ for each $\xi<o(r)$. If $r$ is an arrangement of $s$ and $o(r)=o(s)$, then we call $r$ a "permutation" of $s$. Unfortunately this notation conflicts with that introduced in [3], but in later papers [4], [5] we found the present terminology more useful.

Following the notation of [5], we define for a given sequence $s$ the sets $P^{a}(s)$ and $P(s)$ to be $\{r ; r$ is an arrangement of $s\}$ and $\{r ; r$ is a permutation of $s\}$ respectively. Furthermore, we put $S(s)=\{\Sigma(r) ; r \in P(s)\}$, and define $S^{a}(s)$ similarly, where for any sequence $t, \Sigma(t)$ is the sum of the associated series: $\Sigma(t)=t_{0}+t_{1}+\cdots+t_{\xi}+\ldots, \xi<o(t)$.

In [4], [5] we were interested in obtaining precise upper bounds for $|S(s)|$ as $s$ ranges over all $\alpha$-sequences, $\alpha$ being a fixed ordinal. Here of course "| " denotes cardinality. In this paper we are interested in determining necessary and sufficient conditions on a sequence $s$ in order that $|S(s)|=1$; it seems natural to call such a sequence "commutative". If we have $\left|S^{a}(s)\right|=1$, then we shall say that $s$ is $A$-commutative; clearly $A$-commutativity implies commutativity.

Given two sequences $r, s$, we define the sequence $t=r$ e $s$ as follows. We have $o(t)=o(r), t_{\xi}=r_{\xi}$ for $\xi<o(r)$, and $t_{o(r)+\zeta}=s_{\zeta}$ for $\zeta<o(s)$. Clearly 
this definition can be extended to any well-ordered set of sequences.

A sequence $r$ is called a "segment" of a sequence $s$ if there exist sequences $u, v$ such that $s=u \bullet r \dot{v} v$ : in addition, $r$ is called "initial" ("final") if $u=\varnothing(v=\varnothing)$. Finally, let $r$ be a subsequence of $s$, and suppose that in this case $r=s[X]$ for some $X \subseteq o(s)$ that has been completely specified. We say that $r$ is cofinal in $s$ if $X$ is cofinal in $o(s)$, that is, for each $\xi<o(s)$ there exists $\zeta \in X$ with $\zeta \geqslant \xi$.

A nonzero ordinal $\rho$ is called a "prime component" if $\tau+\rho=\rho$ for every $\tau<\rho$. It is well known that $\rho$ is a prime component if and only if $\rho=\omega^{\delta}$ for some ordinal $\delta$ : Cantor's famous theorem says that every nonzero ordinal $\alpha$ has a unique representation of the form $\rho_{0} a_{0}+\rho_{1} a_{1}+\cdots+\rho_{n-1} a_{n-1}$, where $\left(\rho_{i}\right)_{i<n}$ is a strictly decreasing sequence of prime components and the $a_{i}$ are positive integers. We shall call this the "prime component representation" (pcr) of $\alpha$, and denote $\rho_{0}, \rho_{n-1}$ by " $E(\alpha)$ ", “ $R(\alpha)$ " respectively. For any sequence $s$ of ordinals, we put $E(s)=\left(E\left(s_{\xi}\right)\right)_{\xi<o(s)}$.

Let $\alpha$ be a nonzero ordinal. By " $\operatorname{cf}(\alpha)$ " we denote the cofinality of $\alpha$, which we define to be the least ordinal $\beta$ such that there exists a function $f: \beta \rightarrow \alpha$ with $f^{\prime \prime} \beta$ cofinal in $\alpha$. Clearly $\operatorname{cf}(\alpha) \leqslant \alpha$, and if $\operatorname{cf}(\alpha)=\alpha$, then $\alpha$ is called "regular"; otherwise $\alpha$ is called "nonregular". An ordinal $\alpha$ is called "initial" if $|\beta|<|\alpha|$ for every $\beta<\alpha$, and it can be shown that all regular ordinals are initial: nonregular initial ordinals are usually called "singular". A sequence $s$ is called "regular" ("singular") if $o(s)$ is regular (singular). We sometimes denote the $(1+\alpha)$ th infinite initial ordinal by " $\omega_{\alpha}$ ", and henceforth whenever we refer to an ordinal as being initial, we shall mean that it is infinite and initial.

We list some results that will be of use to us.

Result 1. Given any two ordinals $\alpha, \beta$, we have $\alpha+\beta=\beta+\alpha$ if and only if $\alpha=\gamma m$ and $\beta=\gamma n$ for some ordinal $\gamma$ and some natural numbers $m$, $n$.

Proved in [7].

RESULT 2. A finite sequence $s$ of ordinals is commutative if and only if there exist a positive ordinal $\gamma$ and positive integers $c_{i}, i<o(s)$, such that $s_{i}=\gamma c_{i}$ for each $i<o(s)$.

Immediate from Result 1 and our convention that sequences of ordinals have only positive terms.

Result 3. Let $\alpha, \beta$ be two ordinals such that $\alpha+\beta=\beta$. Then $\alpha+\xi+\beta$ $=\xi+\beta$ for every ordinal $\xi$.

Proved in [6].

RESULT 4. Let $\alpha$ be an ordinal, let $s$ be a nonempty sequence of ordinals, and let $r$ be a subsequence of $s$ such that $\Sigma(r)+\alpha=\alpha$. Then $\Sigma(s)+\alpha=$ $\Sigma(s-r)+\alpha$.

Proved in [5]. 
LEMMA 1. Let $s$ be a sequence of ordinals such that $\Sigma(s)$ is a prime component, and let $r, t$ be complementary subsequences of $s$. Then $\Sigma(s)=$ $\max \{\Sigma(r), \Sigma(t)\}$.

Proof. Clearly it suffices to show that either $\Sigma(s)=\Sigma(r)$ or $\Sigma(s)=\Sigma(t)$. Thus we put $\Sigma(s)=\sigma$ and suppose that $\Sigma(r) \neq \sigma$. Then of course $\Sigma(r)<\sigma$ and so $\Sigma(r)+\sigma=\sigma$. Hence by Result $4, \sigma 2=\Sigma(s)+\sigma=\Sigma(t)+\sigma$, and now it follows easily that $\Sigma(t)=\sigma$.

LeMMA 2. Let $\rho$ be a prime component, let $s$ be any $\rho$-sequence, and let $r, t$ be complementary subsequences of $s$. Then $\rho=\max \{o(r), o(t)\}$.

Proof. Obviously it suffices to demonstrate the result for one particular $\rho$-sequence, and so we take the $\rho$-sequence $s$ defined by $s_{\xi}=1$ for every $\xi<\rho$. Then for any subsequence $u$ of $s$, we have $\Sigma(u)=o(u)$. Our result now follows immediately from the preceding lemma.

LEMMA 3. Let $r, t$ be complementary subsequences of a sequence $s$ of ordinals, and assume that:

(1) $r$ is cofinal in $s$;

(2) $\Sigma(r)$ is a prime component;

(3) $\Sigma(t) \leqslant \Sigma(r)$

(4) if $\Sigma(t)=\Sigma(r)$, then $t$ is cofinal in $s$.

Then $\Sigma(s)=\Sigma(r)$.

Proof. Put $\rho=\Sigma(r)$; obviously $\Sigma(s) \geqslant \rho$, whence we need only show that $\Sigma(s) \leqslant \rho$, and we claim that this will follow once we prove that $\Sigma\left(s^{\prime}\right)<\rho$ for every proper initial segment $s^{\prime}$ of $s$. This claim is self-evident if $o(s)$ is a limit ordinal; thus suppose that $o(s)=\gamma+1$ for some ordinal $\gamma$. Since $r$ is cofinal in $s$, we must have $o(r)=\delta+1$ for some $\delta$ and $r_{\delta}=s_{\gamma}$. Because $\rho$ is a prime component it follows that $s_{\gamma}=\rho$, and so if $\Sigma\left(s^{\prime}\right)<\rho$ for every proper initial segment $s^{\prime}$ of $s$, then it is clear that $\Sigma(s)=\rho$.

Therefore it remains to show that $\Sigma\left(s^{\prime}\right)<\rho$ for every proper initial segment $s^{\prime}$ of $s$. Suppose that this is not the case; then there is a shortest initial segment $u$ of $s$ such that $\Sigma(u) \geqslant \rho$. Now if $o(u)=\psi+1$ for some $\psi$, then it must be that $u_{\psi}=\rho$. But $r, t$ are complementary in $s$, and so $u_{\psi}$ is a term of exactly one of them. We cannot have $u_{\psi}$ being a term of $r$, for if it were, then because $u$ is proper in $s$ and $r$ is cofinal in $s$, we would have the contradiction $\Sigma(r)=\rho+\theta$ for some ordinal $\theta>0$. Thus $u_{\psi}$ must be a term of $t$, whence $\Sigma(t) \geqslant \rho$. By (3), $\Sigma(t) \leqslant \rho$, and so by (4), $t$ is cofinal in $s$, and we are led to the similar contradiction $\Sigma(t)>\rho$.

Hence $o(u)$ is a limit ordinal, from which it follows that $\Sigma(u)=\rho$. Let $r^{u}$, $t^{u}$ be the initial segments of $r, t$ respectively that are complementary in $u$. Then because $u$ is proper in $s$ and $r$ is cofinal in $s, r^{u}$ must be proper in $r$, whence $\Sigma\left(r^{u}\right)<\Sigma(r)=\rho=\Sigma(u)$. Thus Lemma 1 tells us that $\Sigma\left(t^{u}\right)=\rho$, 
and this, combined with (3), (4), tells us that $t$ is cofinal in $s$. But we are once again faced with the contradictory $\Sigma(t)>\rho$. Therefore no such $u$ can exist, and so $\Sigma\left(s^{\prime}\right)<\rho$ for every proper initial segment $s^{\prime}$ of $s$.

Lemma 4. Let $\kappa$ be an initial ordinal, and take any $\delta$ with $0<\delta \leqslant \kappa$. For each $\xi<\delta$ let $s^{\xi}$ be any $\kappa$-sequence. Then there exists a $\kappa$-sequence $s$ containing the $s^{\xi}$ as subsequences in such a way that there exists a partitioning $\left\{X_{\xi} ; \xi<\delta\right\}$ of $\kappa$ with $s^{\xi}=s\left[X_{\xi}\right]$ for every $\xi<\delta$.

We omit the proof of this, because it really is just a "manual-labour" consequence of the fact that $|\delta||\kappa|=|\kappa|$ for every $\delta$ with $0<\delta \leqslant \kappa$.

Let $F$ be a functional from the class of all sequences of ordinals into the class of ordinals, and let $s$ be a sequence. We shall say that the ordinal $\alpha=F(s)$ is an invariant of $s$ if we have $\alpha=F(t)$ for every permutation $t$ of $s$. As an obvious example, $o(s)$ is an invariant of $s$ for every sequence $s$.

Similarly, if $\Gamma$ is a unary predicate with domain the class of all sequences of ordinals, then we say that $\Gamma$ is an invariant of the sequence $s$ if $\Gamma(s) \Leftrightarrow \Gamma(t)$ for every permutation $t$ of $s$. For any given ordinal $\alpha$ and each sequence $s$, the predicate $\Gamma$ defined by $\Gamma(s) \equiv s[=\alpha]=\varnothing$ is an invariant of $s$.

Let $s$ be an $\omega$-sequence of ordinals. Sierpiński showed in [6] that if $s$ is increasing, then $s$ is commutative; later, Ginsburg extended this result to all regular sequences. However, it takes very little thought to see that in neither of these cases is the condition of monotonicity a necessary one for commutativity; we need only consider the $\omega$-sequence $s$ given by $s_{0}=\omega$ and $s_{n}=n$ for all $n>0$.

Whilst the Sierpiński-Ginsburg condition is a very nice one, it has the disadvantage of not being an invariant of the sequence concerned. We wish to present a condition which, although weaker than monotonicity, is still sufficient for commutativity and is an invariant.

Let $s$ be a sequence of ordinals. We say that $s$ is semimonotone if for every pair $(\alpha, \beta)$ of ordinals with $\alpha, \beta<o(s)$, there exists an ordinal $\gamma<o(s)$ such that $\alpha \leqslant \gamma$ and $s_{\beta} \leqslant s_{\gamma}$. Clearly every increasing sequence is semimonotone but not conversely.

THEOREM 1. Semimonotonicity is invariant with respect to regular sequences.

Proof. Let $s$ be a semimonotone $\kappa$-sequence, where $\kappa$ is a regular ordinal, let $t$ be any permutation of $s$, and let $f: \kappa \rightarrow \kappa$ be any bijection such that $t_{\xi}=s_{f(\xi)}$ for each $\xi<\kappa$.

Take any pair of ordinals $\alpha, \beta<\kappa$, and put $\psi=f(\beta)$; then $s_{\psi}=t_{\beta}$. Since $s$ is semimonotone, the set $X=\left\{\xi<\kappa ; s_{\xi} \geqslant s_{\psi}\right\}$ must be cofinal in $\kappa$, and so $|X|=|\kappa|$, by regularity. But then $\left|f^{-1} X\right|=|\kappa|$, and so $f^{-1} X$ is cofinal in $\kappa$.

In particular, then, there must be $\theta<\kappa$ such that $\alpha \leqslant \theta$ and $f(\theta) \in X$. For 
any such $\theta$ we have $t_{\theta}=s_{f(\theta)} \geqslant s_{\psi}=t_{\beta}$. Hence $t$ is semimonotone.

Our next result is proved in a manner similar to the Ginsburg method.

THEOREM 2. Every regular, semimonotone sequence is commutative.

Proof. Let $s$ be such a sequence and put $\kappa=o(s)$. Let $t$ be a permutation of $s$; we shall define a $\kappa$-subsequence $r$ of $t$ with the property that $s_{\xi} \leqslant r_{\xi}$ for all $\xi<\kappa$. It will follow that $\Sigma(s) \leqslant \Sigma(t)$, and symmetry considerations will give us the reverse inequality.

Let $f: \kappa \rightarrow \kappa$ be a bijection such that $s_{\xi}=t_{f(\xi)}$ for each $\xi<\kappa$. We shall define by transfinite induction an increasing function $g: \kappa \rightarrow \kappa$ such that the $\kappa$-subsequence $r=\left(t_{g(\xi)}\right)_{\xi<\kappa}$ of $t$ has the desired property.

Since $s$ is semimonotone, there exists $\gamma<\kappa$ such that $\gamma \geqslant 1$ and $s_{\gamma} \geqslant s_{0}$; let $\alpha$ be the least such $\gamma$ and put $g(0)=f(\alpha)$. Then we have $s_{0} \leqslant s_{\alpha}=t_{g(0)}$.

Assume now that for some $\theta<\kappa$ we have defined $g(\xi)$ for all $\xi<\theta$ in such a manner that $g(\xi)<g(\zeta)$ for all $\xi$, $\zeta$ with $\xi<\zeta<\theta$ and $s_{\xi} \leqslant t_{g(\xi)}$ for all $\xi<\theta$. Since $\kappa$ is regular, $g^{\prime \prime} \theta$ cannot be cofinal in $\kappa$; let $\psi<\kappa$ be such that $g^{\prime \prime} \theta \subseteq \psi$ and consider the pair $(\psi, f(\theta))$. By Theorem 1 the sequence $t$ is semimonotone, and so there exists $\gamma<\kappa$ with $\gamma \geqslant \psi$ and $t_{\gamma} \geqslant t_{f(\theta)}$. We define $g(\theta)$ to be the least such $\gamma$. Then we have $g(\xi)<\psi \leqslant g(\theta)$ for all $\xi<\theta$, and $s_{\theta}=t_{f(\theta)} \leqslant t_{g(\theta)}$.

By induction therefore we have our function $g: \kappa \rightarrow \kappa$, and hence the required sequence $r$. Clearly $\Sigma(s) \leqslant \Sigma(r) \leqslant \Sigma(t)$, and a completely symmetric argument establishes the reverse inequality.

Whilst the preceding result constitutes a slight improvement on Ginsburg's theorem, it still does not provide a necessary condition for commutativity, as the example given earlier shows.

We conclude this section with a necessary and sufficient condition for the $A$-commutativity of a sequence.

THEOREM 3. A sequence $s$ is $A$-commutative if and only if $o(s)$ is finite and there exists an ordinal $\gamma>0$ such that for each $k<o(s)$ we have $s_{k}=\gamma c_{k}$ for some number $c_{k}>0$.

Proof. Obviously for finite sequences the concepts of $A$-commutativity and commutativity are equivalent. In view of Result 2 then, our result will be proved once we show that no sequence of infinite length is $A$-commutative.

Suppose then that $s$ is an $\alpha$-sequence, where $\alpha$ is an infinite ordinal. We claim that there exists an ordinal $\delta>0$ such that for some $\alpha$-sequence $t$ we have $s_{\xi}=\delta t_{\xi}$ for each $\xi<\alpha$, and $t_{\zeta}=\psi+1$ for some $\zeta<\alpha$ and some ordinal $\psi$.

Put $\delta=\min \left\{R\left(s_{\xi}\right) ; \xi<\alpha\right\}$, then $\delta$ is a prime component, and from properties of prime components and the right distributive law we see that for 
each $\xi<\alpha$ we have $s_{\xi}=\delta t_{\xi}$ for some ordinal $t_{\xi}>0$. Then the leftcancellation law ensures that each $t_{\xi}$ is uniquely determined, and so we have our ordinal $\delta$ and our $\alpha$-sequence $t=\left(t_{\xi}\right)_{\xi<\alpha}$. Take any $\zeta<\alpha$ for which $R\left(s_{\zeta}\right)=\delta$; then $t_{\zeta}$ must be a successor ordinal. Thus $t$ has the required properties, and from the set $\left\{s_{\xi}=\delta t_{\xi}\right\}_{\xi<\alpha}$ of equations and the right distributive law it is a simple matter to show that $S^{a}(s)=\left\{\delta \tau ; \tau \in S^{a}(t)\right\}$. Hence by cancellation, $s$ is $A$-commutative if and only if $t$ is $A$-commutative.

Let $\zeta$ be such that $t_{\zeta}$ is a successor ordinal. Since $\alpha$ is infinite, there exists an arrangement $u$ of $t$ such that $o(u)=\theta+1$ for some $\theta$ and $u_{\theta}=t_{\zeta}$. It follows of course that $\Sigma(u)$ is a successor ordinal. Using again the fact that $\alpha$ is infinite, we now obtain an arrangement $v$ of $t$ such that $o(v)$ is a (nonzero) limit ordinal, and from this and the fact that each $t_{\xi}$ is nonzero we conclude that $\Sigma(v)$ is a limit ordinal. Thus $\Sigma(u) \neq \Sigma(v)$, and so $t$ is not $A$-commutative. Hence $s$ is not $A$-commutative.

2. In this section we shall present a necessary and sufficient condition for the commutativity of a regular sequence. This criterion, as was mentioned earlier, will be expressed with the aid of a certain invariant, and our first task is to find the appropriate invariant.

The ordinal in question has already been defined and shown to be an invariant in a special case by Sierpiński; later, Ginsburg showed that the invariance could be extended to the general case.

Let $s$ be an $\omega$-sequence. Sierpiński showed in [6] that $R(\Sigma(s))$ is an invariant of $s$ : this result was extended by Ginsburg in [2] to all regular sequences. Since it is of considerable importance to us, and since Ginsburg simply indicated the proof of the general result, we give the full argument below.

THEOREM 4 (SIERPIŃSKI-GINSBURG). Let $s$ be a regular sequence, and put $\sigma=R(\Sigma(s))$. Then $R(\Sigma(t))=\sigma$ for each permutation $t$ of $s$.

Proof. Put $\kappa=o(s)$ and $\tau=R(\Sigma(t))$. In view of the symmetry of the situation, it is clearly sufficient to show that $\tau \leqslant \sigma$.

Let $u$ be some final segment of $s$ such that $\Sigma(u)=\sigma$. Then $o(s-u)<\kappa$ and so by regularity there must be some final segment $w$ of $t$ such that $\Sigma(w)=\tau$ and some arrangement $r$ of $s-u$ is a subsequence of $t-w$. But then some permutation of $w$ must be a subsequence of $u$, and therefore, because $o(u)$ is a limit ordinal, we must have $w_{\xi}<\sigma$ for every $\xi<o(w)=\kappa$.

In particular, $w_{0}<\sigma=\Sigma(u)$, and so there must be a proper initial segment $u^{0}$ of $u$ such that $w_{0} \leqslant \Sigma\left(u^{0}\right)$. Suppose now that for some $\delta<\kappa$ and for each $\zeta<\delta$ we have defined a segment $u^{\zeta}$ of $u$ in such a way that $w_{\zeta} \leqslant \Sigma\left(u^{\zeta}\right)$ and

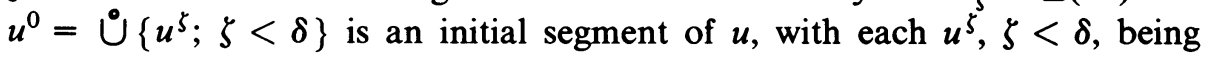
nonfinal in $u$. Since $\kappa$ is regular, we have $o\left(u^{0}\right)<\kappa$, whence $\Sigma\left(u-u^{0}\right)=\sigma$. 
Thus $u-u^{0}$ has a proper initial segment $u^{\delta}$ such that $w_{\delta} \leqslant \Sigma\left(u^{\delta}\right)$.

Hence by induction we obtain such a segment $u^{\xi}$ of $u$ for each $\xi<\kappa$, and so $\tau=\Sigma(w) \leqslant \Sigma\left\{\Sigma\left(u^{\xi}\right) ; \xi<\kappa\right\} \leqslant \Sigma(u)=\sigma$, as required.

In this and to a certain extent in the following sections we shall make use of a certain type of ordinal subtraction. Let $\alpha$ be a nonzero ordinal with pcr $\rho_{0} a_{0}+\cdots+\rho_{n-1} a_{n-1}$; then for any prime component $\rho$ we define $a \circ \rho$ to be $\rho_{0} a_{0}+\cdots+\rho_{k} a_{k}$, where $k$ is the largest number $i<n$ such that $\rho_{i} \geqslant \rho$, if such exist; otherwise we define $a \circ \rho$ to be $\alpha$. Our motivation for this definition resides in the easily proved fact that for any prime component $\rho$ and any two ordinals $\alpha, \beta \geqslant \rho$, we have $\alpha+\beta+\rho=\beta+\alpha+$ $\rho$ if and only if $\alpha \stackrel{\circ}{\rho}+\beta \stackrel{\circ}{\circ}=\beta \stackrel{\circ}{\rho}+\alpha \stackrel{\circ}{\circ}$.

If $s$ is any sequence and $\rho$ any prime component, then we define the sequence $s \circ \rho$ to be $t$, where $o(t)=o(s)$ and $t_{\xi}=s_{\xi} \circ \rho$ for each $\xi<o(s)$ : clearly all terms of $s \stackrel{\circ}{\rho}$ are nonzero. If-and only if $-s$ is a regular sequence and $\rho=R(\Sigma(s))$, then we denote $s \circ \rho$ by " $C(s)$ ".

Given any sequence $s$ and any bijection $f: o(s) \rightarrow o(s)$, then we denote by " $f[s]$ " the permutation $t$ of $s$ defined by $s_{\xi}=t_{f(\xi)}, \xi<o(s)$.

LEMMA 5. Let $s$ be a $\kappa$-sequence, with $\kappa$ a regular ordinal.

(1) $o(s[\geqslant \sigma])=o(C(s)[\geqslant \sigma])<\kappa$, where $\sigma=R(\Sigma(s))$;

(2) $C(f[s])=f[C(s)]$ for each bijection $f: \kappa \rightarrow \kappa$.

Proof. (1) For any nonzero ordinal $\alpha$ and prime component $\rho$, we obviously have $\alpha \geqslant \rho$ if and only if $\alpha \circ \rho \geqslant \rho$. Thus $o(s[\geqslant \sigma])=$ $o(C(s)[\geqslant \sigma])$, and it suffices to show that $o(s[\geqslant \sigma])<\kappa$.

Suppose that $o(s[\geqslant \kappa])=\kappa$. Since $\kappa$ is initial, it follows that $\Sigma(u) \geqslant \sigma \kappa>\sigma$ for every nonempty final segment $u$ of $s$. But it follows from the definition of $\sigma$ and the fact that $o(s)$ is a limit ordinal that we must have $\Sigma(u)=\sigma$ for some nonempty final segment $u$ of $s$. Thus it must be the case that $o(s[\geqslant \sigma])$ $<\kappa$.

(2) This follows at once from Theorem 4 and the definitions.

Our first task is to show that as far as commutativity is concerned, we can without loss of generality cut off the "tails" of all terms of a regular sequence $s$ and assume that $s=C(s)$. We commence with the following result.

THEOREM 5. Let $s$ be a regular sequence. Then $\Sigma(C(s))=\Sigma(s)$.

Proof. Put $\kappa=o(s), \sigma=R(\Sigma(s)), t=C(s), r=s[\geqslant \sigma], u=t[\geqslant \sigma], \gamma=$ $o(r)$. We can express $\gamma$ uniquely as $\theta+k$, where $\theta$ is a limit ordinal (possibly 0 ) and $k$ is a natural number. We let $r^{\#}$ be the initial segment of $r$ having length $\theta$, and define $u^{\#}$ similarly with respect to $u$. Furthermore, we let $s^{\#}$ be the initial segment of $s$ in which $r^{\#}$ is cofinal, and define $t^{\#}$ similarly with respect to $t$ and $u^{\#}$. 
We wish to show firstly that $\Sigma\left(s^{\#}\right)-\Sigma\left(t^{\#}\right)$. Take any $\zeta<\theta$ and let $s^{\zeta}$ be

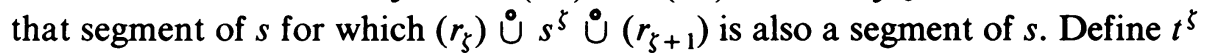
similarly with respect to $u_{\zeta}$ and $u_{\zeta+1}$. From the definition of $t$ and $u$ we see that $r_{\xi}=u_{\zeta}+\mu$ for some $\mu$ such that $\mu+r_{\zeta+1}=r_{\zeta+1}$. Hence from Result 3 we see that $r_{\zeta}+\Sigma\left(s^{\zeta}\right)+r_{\zeta+1}=u_{\zeta}+\Sigma\left(s^{\zeta}\right)+r_{\zeta+1}$. But of course $s^{\zeta}=t^{\zeta}$, and so $r_{\zeta}+\Sigma\left(s^{\zeta}\right)+r_{\zeta+1}=u_{\zeta}+\Sigma\left(t^{\zeta}\right)+r_{\zeta+1}$, from which it follows by a simple induction argument that

$$
\sum\left\{r_{\zeta+m}+\sum\left(s^{\zeta+m}\right) ; m<\omega\right\}=\sum\left\{\mu_{\zeta+m}+\sum\left(t^{\zeta+m}\right) ; m<\omega\right\} .
$$

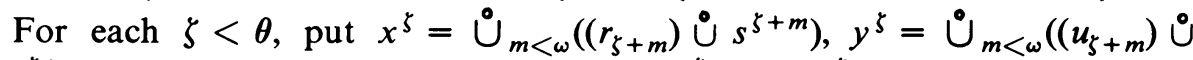
$\left.t^{\zeta+m}\right)$ : then it has just been shown that $\Sigma\left(x^{\zeta}\right)=\Sigma\left(y^{\zeta}\right)$. But $\theta=\omega \tau$ for some $\tau$, and it is clear that $s^{\#}=\dot{\cup}_{\psi<\tau} x^{\omega \psi}, t^{\#}=\cup_{\psi<\tau} y^{\omega \psi}$. Thus $\Sigma\left(s^{\#}\right)=\Sigma\left(t^{\#}\right)$, as desired.

Now if $\gamma=\theta$, then our result follows immediately. Thus we may assume that $\gamma=\alpha+1$ for some $\alpha$, and we let $s^{\prime}, s^{\prime \prime}$ be segments of $s$ such that $s=s^{\#} \cup s^{\prime} \cup\left(r_{\alpha}\right) \cup s^{\prime \prime}$ : we define segments $t^{\prime}, t^{\prime \prime}$ of $t$ similarly. Then precisely the same inductive argument as before shows that $\Sigma\left(s^{\prime}\right)+r_{\alpha}=$ $\Sigma\left(t^{\prime}\right)+r_{\alpha}$. However, it is easy to see that $\Sigma\left(s^{\prime \prime}\right)=\Sigma\left(t^{\prime \prime}\right) \geqslant \sigma$, whence we have $r_{\alpha}+\Sigma\left(s^{\prime \prime}\right)=u_{\alpha}+\Sigma\left(s^{\prime \prime}\right)=u_{\alpha}+\Sigma\left(t^{\prime \prime}\right)$.

Therefore $\Sigma(s)=\Sigma(t)$.

Corollary. Let $s$ be a regular sequence. Then $S(s)=S(C(s))$.

Proof. We apply the preceding result to Lemma 5(2).

It is obvious from this corollary that if $s$ is a regular sequence, then $s$ is commutative if and only if $C(s)$ is commutative. Hence for our purposes it suffices to deal with regular sequences $s$ for which $s=C(s)$. We call such sequences "homogeneous".

THEOREM 6. Let $s$ be a homogeneous sequence, and put $\sigma=R(\Sigma(s))$. Then $|S(s)| \geqslant\left|S^{a}(s[\geqslant \sigma])\right|$.

Proof. Put $\kappa=o(s), r=s[\geqslant \sigma]$, and $t=s-r$. From Lemma 5(1) we have $o(r)<\kappa$; it follows that if $t^{\prime}, t^{\prime \prime}$ are any segments of $t$ with $t^{\prime \prime} \neq \varnothing$ and $t^{\prime} \cup t^{\prime \prime}=t$, then $t^{\prime} \cup \dot{0} \cup t^{\prime \prime} \in P(s)$ for each $u \in P^{a}(r)$. Furthermore, $r$ cannot be cofinal in $s$, whence there exists a final segment $t^{\prime \prime}$ of $t$ such that $\Sigma\left(t^{\prime \prime}\right)=\sigma=R(\Sigma(t))$. Thus $\Sigma(t)=\alpha+\sigma$ for some $\alpha$, and we may without loss of generality assume that $\Sigma\left(t^{\prime}\right) \geqslant \alpha$, where $t^{\prime}=t-t^{\prime \prime}$.

Combining this with the preceding observation, we see that for each $\beta \in S^{a}(r)$ we have $\gamma+\beta+\sigma \in S(s)$, where $\gamma=\Sigma\left(t^{\prime}\right)$. We are however assuming that $s$ is homogeneous, and so we must have $R\left(r_{\xi}\right) \geqslant \sigma$ for each $\xi<o(r)$, whence it follows easily that $R(\beta) \geqslant \sigma$ for each $\beta \in S^{a}(r)$. Therefore if $\beta, \delta \in S^{a}(r)$ are such that $\beta \neq \delta$, then $\beta+\sigma \neq \delta+\sigma$, and so of course $\gamma+\beta+\sigma \neq \gamma+\delta+\sigma$. 
Thus $|S(s)| \geqslant\left|S^{a}(r)\right|$.

THEOREM 7. Let $s$ be a regular sequence, put $\sigma=R(\Sigma(s))$, and assume that $s[\geqslant \sigma]=\varnothing$. Then $s$ is commutative and $S(s)=\{\sigma\}$.

Proof. It is easily proved that $\operatorname{cf}(\sigma)=\operatorname{cf}(\kappa)=\kappa$, where $\kappa=o(s)$. Take any permutation $t$ of $s$; we know that $R(\Sigma(t))=\sigma$, and so if $\Sigma(t) \neq \sigma$, then we must have $\Sigma(t)=\alpha+\sigma$ for some ordinal $\alpha \geqslant \sigma$. Suppose this to be the case; then we certainly have $\Sigma\left(t^{\prime}\right) \geqslant \sigma$ for some proper initial segment $t^{\prime}$ of $t$, and we let $u$ be the shortest (with respect to length) of these. If $o(u)$ is a limit ordinal, then an ordinary limit argument gives $\Sigma(u)=\sigma$, which in turn yields the contradictory $\operatorname{cf}(\sigma)=\operatorname{cf}(o(u)) \leqslant o(u)<\kappa$. Thus we must have $o(u)=\gamma$ +1 for some $\gamma$. But then $\Sigma\left(\left(u_{\xi}\right)_{\xi<\gamma}\right)<\sigma$ by definition of $u$, and $u_{\gamma}<\sigma$ by the assumption $s[\geqslant \sigma]=\varnothing$. Thus we are led to a second contradiction, $\Sigma(u)<\sigma$. Therefore our supposition regarding $t$ was incorrect, and our result is proved.

Combining these results, we obtain our desired criterion.

THEOREM 8. Let $s$ be a regular sequence, and put $\sigma=R(\Sigma(s)), r=$ $C(s)[\geqslant \sigma]$. Then $s$ is commutative if and only if $o(r)$ is finite and there exists an ordinal $\gamma>0$ such that, for each $k<o(r), r_{k}=\gamma c_{k}$ for some number $c_{k}>0$.

Proof. The necessity of the condition follows at once from Theorem 5 corollary, Theorem 6, and Result 2 . Therefore we assume that the condition holds and put $n=o(r)$. We claim that $\Sigma(s)=\Sigma(r)+\sigma$, and by Theorem 5 it suffices to show that $\Sigma(C(s))=\Sigma(r)+\sigma$. Therefore we may assume without loss of generality that $s=C(s)$, whence of course $r$ is a subsequence of $s$.

Put $u=s-r$; since $r$ is finite, some nonempty final segment of $s$ must also be nonempty and final in $u$, whence $R(\Sigma(u))=\sigma$. Since $u[\geqslant \sigma]=\varnothing$, Theorem 7 tells us that $u$ is commutative and that $\Sigma(u)=\sigma$.

For each $i \leqslant n$, let $u^{i}$ be that segment of $u$ for which $u=\cup_{i<n}\left(u^{i} \cup\left(r_{i}\right)\right)$ $\stackrel{\bullet}{u^{n}}$. Then $u^{n}$ is a nonempty final segment of $u$ and each $u^{i}$ with $i<n$ is a nonfinal segment of $u$. Hence $\Sigma\left(u^{i}\right)<\sigma$ for each $i<n$ and $\Sigma\left(u^{n}\right)=\sigma$. But now a repeated application of Result 3 tells us that $\Sigma(s)=\Sigma(r)+\sigma$.

Let $t$ be a permutation of $s$, and put $v=C(t)[\geqslant \sigma]$. By Theorem 4 we have $R(\Sigma(t))=\sigma$, and so Lemma $5(2)$ and the finiteness of $o(r)$ tell us that $v$ is a permutation of $r$. By the same argument as above we arrive at $\Sigma(t)=\Sigma(v)+$ $\sigma$. But $r$ is commutative, and so $\Sigma(t)=\Sigma(s)$. This gives us our result.

3. In the preceding section we considered commutativity of regular sequences: in this we shall deal with singular sequences. As in the previous case, our first task is to find an appropriate invariant.

Let $s$ be any nonempty sequence; then clearly there exist ordinals $\theta$ such that $|o(s[>\theta])|<|o(s)|$, and we define $M(s)$ to be the least such $\theta$. 
Obviously $M(s)$ is an invariant of $s$, and it is easily seen that for each $\gamma<M(s)$ we have $|o(s[>\gamma, \leqslant M(s)])|=|o(s)|$.

We say that $s$ is bounded if $s[>M(s)]=\varnothing$; in this section bounded sequences play much the same role as did homogeneous sequences in the last.

THEOREM 9. Let $s$ be a bounded singular sequence, and put $\kappa=o(s)$, $\theta=M(s)$. Then $\Sigma(r)=\theta \kappa$ for some permutation $r$ of $s$.

PROof. Since $s$ is bounded, we have $\Sigma(r) \leqslant \theta \kappa$ for every permutation $r$ of $s$. Therefore it suffices to show that $\Sigma(r) \geqslant \theta \kappa$ for some such $r$.

Let us dispose of three simple cases. If $o(s[=\theta])=\kappa$, then clearly $\Sigma(s) \geqslant$ $\theta \kappa$. If $\theta<\kappa$, then $\kappa \leqslant \Sigma(s) \leqslant \theta \kappa=\kappa$, and so again we conclude that $\Sigma(s)=$ $\theta \kappa$. Finally, if $\kappa^{\alpha}<\theta<\kappa^{\alpha+1}$ for some $\alpha$, then by definition of $\theta$ we have $o\left(s\left[\geqslant \kappa^{\alpha}\right]\right)=\kappa$, whence $\kappa^{\alpha+1}=\kappa^{\alpha} \kappa \leqslant \Sigma(s) \leqslant \theta \kappa=\kappa^{\alpha+1}$, and thus $\Sigma(s)=$ $\kappa^{\alpha+1}=\theta \kappa$.

We may therefore assume that $o(s[=\theta])<\kappa$ and that $\theta=\kappa^{\alpha}$ for some $\alpha>0$. Put $t=s[<\theta]$; since $o(s[=\theta])<\kappa$, it follows from Lemma 2 that $o(t)=\kappa$. Clearly it suffices, to prove our result in this case, to show that $\Sigma(r) \geqslant \theta \kappa$ for some permutation $r$ of $t$.

Now $\theta=\sup \left\{t_{\xi} ; \xi<\kappa\right\}$ : hence if we put $\eta=\operatorname{cf}(\theta)$, then $\eta \leqslant \operatorname{cf}(\kappa)<\kappa$. But $M(t)=\theta>t_{\xi}$ for each $\xi<\kappa$, and so there certainly exists an increasing subsequence $u^{0}$ of $t$ with length $\eta$ and limit $\theta$; let $X_{0}$ be a given subset of $\kappa$ such that $u^{0}=t\left[X_{0}\right]$.

The idea is to define $\kappa$ such subsequences of $t$ and string them "end to end"; in this way we will finish up with a permutation $r$ of $t$ with $\Sigma(r) \geqslant \theta \kappa$. Thus we suppose that $\psi<\kappa$ has been given and that for each $\zeta<\psi$ we have obtained a subset $X_{\zeta}$ of $\kappa$ such that $X_{\xi} \cap X_{\zeta} \neq \varnothing$ for all $\xi, \zeta<\psi$ with $\xi \neq \zeta$, and that for each $\zeta<\psi, t\left[X_{\zeta}\right]$ is an increasing $\eta$-sequence with limit $\theta$. Put $u^{\zeta}=t\left[X_{\zeta}\right], Y=\cup\left\{X_{\zeta} ; \zeta<\psi\right\}$. Since $\psi<\kappa$ and $\left|X_{\zeta}\right|=|\eta|<|\kappa|$ for each $\zeta<\psi$, we see that $|\kappa-Y|=|\kappa|$, whence $o(t[\kappa-Y])=\kappa$. Furthermore, since $|Y|<|\kappa|$, it follows from the definition of $\theta$ that $M(t[\kappa-Y])=\theta$, whence there exists $X_{\psi} \subseteq \kappa-Y$ such that $t\left[X_{\psi}\right]$ is an increasing $\eta$-sequence with limit $\theta$.

Thus by transfinite induction, subsets $X_{\zeta}$ with the desired properties exist

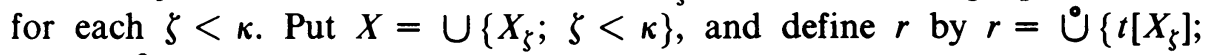
$\zeta<\kappa\} \stackrel{\cup}{t} t \kappa-X]$. Then $r$ is a permutation of $t$, and $\Sigma(r) \geqslant \theta \kappa$.

THEOREM 10. Let $s$ be a bounded singular sequence, and put $\kappa=o(s)$, $\theta=M(s)$. Then $s$ is commutative if and only if at least one of the three following conditions holds:

(1) $\theta<\kappa$;

(2) $\kappa^{\alpha}<\theta<\kappa^{\alpha+1}$ for some ordinal $\alpha>0$;

(3) $o(s[=\theta])=\kappa$. 
Proof. We note that the three conditions listed correspond to the three cases dealt with in the early section of the proof of Theorem 9. Thus we can take any permutation $r$ of $s$ and repeat the arguments given there to show that $\Sigma(r)=\theta \kappa$, since the ordinal $\theta$ and the predicate $o(s[=\alpha])=\kappa(\alpha$ any given ordinal) are invariants of $s$. This shows that the conditions are sufficient for commutativity. Now Theorem 9 tells us that whether or not $s$ satisfies any of the conditions, there is a permutation $r$ of $s$ with $\Sigma(r)=\theta \kappa$. Thus in order to complete the proof it suffices to show that if $s$ fails all three conditions, then there is a permutation $r^{\prime}$ of $s$ such that $\Sigma\left(r^{\prime}\right)=\theta \gamma$ for some ordinal $\gamma<\kappa$. Hence we suppose that $\theta=\kappa^{\alpha}$ for some $\alpha>0$ and that $o(s[=\theta])<$ $\kappa$.

Put $t=s[<\theta]$; by Lemma 2 we have $o(t)=\kappa$, and so $s[=\theta] \cup ீ$ is a permutation of $s$ for each permutation $u$ of $t$. But $\Sigma(s[=\theta])<\theta \kappa$, and so it suffices to show that there is some permutation $u$ of $t$ and some ordinal $\gamma<\kappa$ such that $\Sigma(u)=\theta \gamma$.

Put $\eta=\operatorname{cf}(\theta)$ and $\lambda=\operatorname{cf}(\kappa)$; borrowing once again from the proof of Theorem 9, we see that $\eta \leqslant \lambda<\kappa$. We divide our argument into two cases, the first in which $\eta=\lambda$, and the second in which $\eta<\lambda$.

Assume that $\eta=\lambda$ and let $\left(\theta_{\psi}\right)_{\psi<\eta}$ be an increasing $\eta$-sequence of ordinals with limit $\theta$. Put $t^{0}=t\left[\leqslant \theta_{0}\right]$, and for each $\psi$ with $0<\psi<\eta$, put $t^{\psi}=$ $t\left[>\delta_{\psi}, \leqslant \theta_{\psi}\right]$, where $\delta_{\psi}=\lim _{\zeta<\psi} \theta_{\zeta}$. We now subdivide our argument into two further cases.

In the first of these we suppose that $o\left(t\left[<\theta_{\psi}\right]\right)<\kappa$ for each $\psi<\eta$, whence it follows that $o\left(t^{\psi}\right)<\kappa$ for each such $\psi$. Put $u=\cup_{\psi<\eta} t^{\psi}$; we claim that $u$ is a permutation of $t$ with $\Sigma(u)=\theta$.

Certainly $u$ is an arrangement of $t$, and as $\kappa$ is initial we must have $o(u) \geqslant \kappa$. Suppose that $o(u)>\kappa$ : then $\kappa=\sum_{\xi<\psi} \bar{o}\left(t^{\xi}\right)+\beta$ for some $\psi<\eta$ and some $\beta<o\left(t^{\psi}\right)$. But in view of the definitions of $\eta$ and $\lambda$, this is impossible. Thus $o(u)=\kappa$ and $u$ is a permutation of $t$; it remains to show that $\Sigma(u)=\theta$. Let $u^{\prime}$ be a proper initial segment of $u$; then for some $\psi<\eta$ and some proper initial segment $r$ of $t^{\psi}$ we have $u^{\prime}=\left(\cup_{\xi<\psi} t^{\xi}\right) \stackrel{\bullet}{ } r$. Therefore $u_{\zeta}^{\prime} \leqslant \theta_{\psi}$ for each $\zeta<o\left(u^{\prime}\right)$, and so $\Sigma\left(u^{\prime}\right) \leqslant \theta_{\psi} o\left(u^{\prime}\right)$. Now $\theta_{\psi}<\theta=\kappa^{\alpha}$ for some $\alpha>0$, whence $\theta_{\psi} \kappa \leqslant \theta$. Since $o\left(u^{\prime}\right)<\kappa$, we see that $\Sigma\left(u^{\prime}\right)<\theta$. Thus by the arbitrary choice of $u^{\prime}$ we conclude that $\Sigma(u) \leqslant \theta$. The reverse inequality is clear.

In our second subcase we assume that $o\left(t\left[<\theta_{\psi}\right]\right)=\kappa$ for some $\psi<\eta$, and so $o\left(t^{\psi}\right)=\kappa$ for some such $\kappa$. Define $X \subseteq \eta$ by $X=\left\{\psi<\eta\right.$;o(t $\left.\left.t^{\psi}\right)=\kappa\right\}$ : we once more consider two possibilities.

Suppose that $|X|<|\eta|$. Lemma 4 tells us that there is a $\kappa$-sequence $t^{0}$ containing the $\kappa$-sequences $t^{\psi}$ for $\psi \in X$ as "disjoint" subsequences, and such that each $t_{\xi}^{0}$ occurs in one such $t^{\psi}$. Put $\tau=\sup _{\xi<\kappa} t_{\xi}^{0}$; then $\tau<\theta$. To see this, 
we simply observe that $\tau \leqslant \sup _{\psi \in X} \theta_{\psi}$ and that if $\mu$ is the order-type of any well-ordering of $X$, then $\mu<\eta=\operatorname{cf}(\theta)$.

Let $v \in P\left(t^{0}\right)$ be such that $v$ is a subsequence of $t$, and put $w=t-v$. From $\tau<\theta=M(t)$ we obtain $o(t[>\tau,<\theta])=\kappa$, and hence $o(w)=\kappa$. But $w$ conforms to the hypotheses of the previous case, and so by repeating the above arguments we can show that there is a permutation $w^{0}$ of $w$ with $\Sigma\left(w^{0}\right)=\theta$. Applying Lemma 4 to $t^{0}$ and $w^{0}$, we obtain a permutation $u$ of $t$ containing $t^{0}, w^{0}$ as complementary subsequences. Clearly $\Sigma\left(t^{0}\right) \leqslant \tau \kappa \leqslant \theta$, and so by Lemma 3 we have $\Sigma(u)=\theta$.

We turn to the case $|X|=|\eta|$, and for convenience we denote the $t^{\psi}$ for $\psi \in X$ by " $r^{\mu}$ ", $\mu<\eta$. We wish to obtain a $\kappa$-sequence $v$ containing each $r^{\mu}$ as a subsequence in such a manner that as $\mu$ increases, the subsequences $r^{\mu}$ get further away from the start of $v$. Formally, we achieve this as follows. Recalling that $\eta=\lambda$, we let $\left(\kappa_{\mu}\right)_{\mu<\eta}$ be an increasing $\eta$-sequence of initial ordinals with limit $\kappa$, and for each $\mu<\eta$ we let $z^{\mu}$ be the $\kappa_{\mu}$-sequence defined by $z_{\xi}=0$ for all $\xi<\kappa_{\mu}$. We then put $v^{\mu}=z^{\mu} \cup r^{\mu}$; each $v^{\mu}$ is thus a $\kappa$-sequence with the first $\kappa_{\mu}$ terms all zero, and we apply Lemma 4 to the set $\left\{v^{\mu} ; \mu<\eta\right\}$, obtaining a $\kappa$-sequence $v^{0}$. Finally, we put $v=v^{0}\lceil>0$ ]. Since $v$ contains each $r^{\mu}$, we must have $o(v)=\kappa$. However, if we take any proper initial segment $v^{\prime}$ of $v$ and any $\mu<\eta$ such that $o\left(v^{\prime}\right)<\kappa_{\mu}$, then because $z^{\mu}$ was a subsequence of $v^{0}$, it must be the case that $r^{\mu}$ is a subsequence of $v-v^{\prime}$; more generally, $r^{\nu}$ is a subsequence of $v-v^{\prime}$ for each $\nu$ with $\mu<\nu<\eta$. This is the property that we desired $v$ to have, and we deduce from it that if $v^{\prime}$ is any proper initial segment of $v$, then $\sup \left\{v_{\xi} ; \xi<o\left(v^{\prime}\right)\right\}<$ $\theta$.

Let $x$ be the permutation of $v$ such that $x$ is a subsequence of $t$, and put $u^{0}=t-x$. This time we have no guarantee that $o\left(u^{0}\right)=\kappa$; nevertheless, the same argument as used previously shows that there exists an arrangement $u$ of $u^{0}$ such that $o(u) \leqslant \kappa$ and $\sup \left\{u_{\xi}^{\prime} ; \xi<o\left(u^{\prime}\right)\right\}<\theta$ for each proper initial segment $u^{\prime}$ of $u$.

If $o(u)<\kappa$, put $w=u \stackrel{0}{\text {, }}$, where $z$ is the $\kappa$-sequence defined by $z_{\xi}=0$ for all $\xi<\kappa$; otherwise put $w=u$. Then $w$ is a $\kappa$-sequence and by Lemma 4 there exists a $\kappa$-sequence $y^{0}$ containing $v$ and $w$ as complementary (cofinal) subsequences. Put $y=y^{0}[>0]$; from the construction of $v$ and $w$ it follows that $y$ is a permutation of $t$. Now clearly $v$ is cofinal in $y$ and $\Sigma(v)=\theta \geqslant$ $\Sigma(u)$. Furthermore, if $u$ is not cofinal in $y$, then $o(u)<\kappa$ and it follows that $\Sigma(u)<\theta$. Thus by Lemma 3 we have $\Sigma(y)=\Sigma(v)=\theta$, since of course $\theta$ is a prime component.

We have just seen that in the case $\eta=\lambda$ there is a permutation $u$ of $t$ with $\Sigma(u)=\theta$, and it follows that in this case $s$ is not commutative. Hence we may turn our attention to the case $\eta<\lambda$. 
We show firstly that there is an increasing $\lambda$-sequence $\left(\kappa_{\xi}\right)_{\zeta<\lambda}$ of singular (initial) ordinals with limit $\kappa$ and such that $\operatorname{cf}\left(\kappa_{\zeta}\right)=\eta$ for each $\zeta<\lambda$. Put $\kappa=\omega_{\beta}$; since $\kappa$ is singular, $\beta$ must be a nonzero limit ordinal with $\operatorname{cf}(\beta)=\lambda$. Obviously $\operatorname{cf}(\beta)=\operatorname{cf}(R(\beta))$, and so $\eta<R(\beta)$. Thus for each $\gamma<\beta$ we must have $\gamma+\eta<\beta$. Let $\left(\beta_{\zeta}\right)_{\zeta<\lambda}$ be an increasing $\lambda$-sequence of ordinals such that $\lim _{\zeta<\lambda} \beta_{\zeta}=\beta$ and $R\left(\beta_{\zeta}\right)>\eta$ for each $\zeta<\lambda$, and put $\tau_{\zeta}=\beta_{\zeta}+\eta$. Then $\left(\tau_{\zeta}\right)_{\zeta<\lambda}$ is also an increasing $\lambda$-sequence having limit $\beta$, and it is clear that if we put $\kappa_{\zeta}=\omega_{\tau_{\zeta}}$, then $\left(\kappa_{\zeta}\right)_{\zeta<\lambda}$ is the desired sequence.

Our next task is to show that there exist pairwise disjoint subsets $X_{\zeta}$ of $\kappa$ for $\zeta<\lambda$ such that $\left|o\left(t\left[X_{\zeta}\right]\right)\right|=\left|\kappa_{\zeta}\right|$ and $M\left(t\left[X_{\zeta}\right]\right)=\theta$. This is proved by induction and we illustrate the induction step, the basis step being clear.

Suppose that we have defined appropriate subsets $X_{\zeta}$ for $\zeta<\psi$, where $\psi<\lambda$ is some given nonzero ordinal. Put $X=\cup_{\zeta<\psi} X_{\zeta}$; since $\psi<\lambda=\operatorname{cf}(\kappa)$ and $\left|X_{\xi}\right|=\left|\kappa_{\xi}\right|<|\kappa|$ for each $\zeta<\psi$, it must be the case that $o(t[\kappa-X])=\kappa$ and $M(t[\kappa-X])=\theta$. Put $u=t[\kappa-X]$; then since $\operatorname{cf}(\theta)=\eta$ we can define quite easily an increasing $\eta$-sequence $\left(\theta_{\mu}\right)_{\mu<\eta}$ of ordinals such that $o\left(u\left[>\theta_{\mu}, \leqslant \theta_{\mu+1}\right]\right)>\kappa_{\psi}$ for each $\mu<\eta$. Thus we can extract from $u\left[>\theta_{\mu}, \leqslant \theta_{\mu+1}\right]$ a $\kappa_{\psi}$-sequence $x^{\mu}$. We apply Lemma 4 to the set $\left\{x^{\mu} ; \mu<\eta\right\}$ and obtain a $\kappa_{\psi}$-sequence $x$ with $M(x)=\theta$. Let $Y \subseteq \kappa-X$ and $v \in P(u)$ be such that $x=v[Y]$. Clearly there is some arrangement $y$ of $x$ such that $y$ is a subsequence of $t$; furthermore, we can select $y$ in such a manner that if $f$ : $o(x) \rightarrow o(y)$ is any bijection satisfying $x_{\xi}=y_{f(\xi)}, \xi<o(x)$, then for some such $f$ we have $f^{\prime \prime} Y \subseteq \kappa-X$. We let $f^{\#}$ be such a bijection and put $X_{\psi}=f^{\# \prime \prime} Y$.

This establishes the existence of the desired sets $X_{\zeta}, \zeta<\lambda$, and for each such $\zeta$ we put $t^{\zeta}=t\left[X_{\xi}\right]$. Since $\left|o\left(t^{\zeta}\right)\right|=\left|\kappa_{\zeta}\right|$, we can select $v^{\zeta} \in P^{a}\left(t^{\zeta}\right)$ such that $o\left(v^{\zeta}\right)=\kappa_{\zeta}$. Furthermore, we put $t^{0}=t\left[\kappa-\cup_{\zeta<\lambda} X_{\zeta}\right]$.

Consider firstly the case in which $o\left(t^{0}\right)<\kappa$. Now for each we have $M\left(v^{\zeta}\right)=\theta, o\left(v^{\zeta}\right)=\kappa_{\zeta}$ and $\operatorname{cf}\left(\kappa_{\zeta}\right)=\eta<\kappa_{\zeta}$. In addition, $\theta=\kappa^{\alpha}=\kappa_{\zeta}^{\kappa \alpha}$ for some $\alpha>0$. Thus, with respect to each $v^{\zeta}$, we find ourselves in the situation dealt with in the case $\eta=\kappa$, and hence can infer that $\Sigma\left(w^{5}\right)=\theta$ for some permutation $w^{\zeta}$ of $v^{\zeta}$. Put $w=t^{0} \cup \cup_{\zeta<\lambda} w^{\zeta}$ : from $o\left(t^{0}\right)<\kappa$ we obtain $w \in P(t)$, and it is easy to see that $\Sigma(w)=\sigma+\theta \lambda$ for some $\sigma$. From this we conclude that $\Sigma(w)<\theta \kappa$, and so $t$, hence $s$, is not commutative.

Finally, suppose that $o\left(t^{0}\right)=\kappa$. Let $\left\{Z_{\zeta} ; \zeta<\lambda\right\}$ be a partitioning of $\kappa$ such that $o\left(t^{0}\left[Z_{\zeta}\right]\right)=\kappa_{\zeta}$, and apply Lemma 4 to obtain a $\kappa_{\xi}$-sequence $u^{\zeta}$ having $v^{\zeta}$ and $t^{0}\left[Z_{\zeta}\right]$ as complementary subsequences. Obviously $M\left(u^{\xi}\right)=\theta$, and we can proceed as before to find a permutation $w^{\zeta}$ of $u^{\zeta}$ with $\Sigma\left(w^{\zeta}\right)=\theta$. But then $w=\dot{U}_{\zeta<\lambda} w^{\zeta}$ is a permutation of $t$ and $\Sigma(w)=\theta \lambda<\theta \kappa$. Once again, therefore, $s$ is not commutative.

Having exhausted all possibilities, we reach the end of our proof. 
THEOREM 11. Let $s$ be a singular sequence and put $\kappa=o(s), \theta=M(s)$, $t=s[\geqslant \theta \kappa] \circ \theta \kappa$. Then $s$ is commutative if and only if the following hold:

(1) $s[\leqslant \theta]$ is commutative;

(2) $t$ is finite and commutative;

(3) $s[>\gamma]$ is finite for some $\gamma<\theta \kappa$.

Proof. Suppose that $s$ is commutative. By definition of $\theta$ we have $o(s[>\theta])$ $<\kappa$, and so $s[>\theta] \stackrel{\bullet}{r}$ is a permutation of $s$ for each $r \in P(s[\leqslant \theta])$. It follows that $s[\leqslant \theta]$ is commutative. But clearly $s[\leqslant \theta]$ is a bounded $\kappa$ sequence with $M(s[\leqslant \theta])=\theta$, and so Theorem 9 tells us that $\Sigma(r)=\theta \kappa$ for some permutation $r$ of $s[\leqslant \theta]$. Thus $\Sigma(s[\leqslant \theta])=\theta \kappa$.

Assume that $s[>\gamma]$ is infinite for each $\gamma<\theta \kappa$, and put $r=s[>\theta,<\theta \kappa]$. Then $r$ must have an increasing subsequence $u$ such that $o(u)=\tau$ for some infinite limit ordinal $\tau$ and $\lim _{\xi<\tau} u_{\xi}=\theta \kappa$. Clearly $\tau<\kappa$, and so we must have $\Sigma(v)=\theta \kappa$ for each nonempty cofinal subsequence $v$ of $u$. Let $u^{0}, u^{1}$ be two complementary, cofinal subsequences of $u$, and put $w=s[\geqslant \theta \kappa] \bullet \bullet r-u$

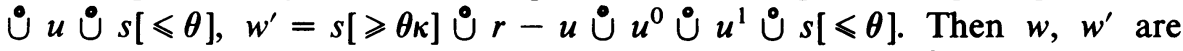
both permutations of $s$ : however, if we put $\sigma=\Sigma(s[\geqslant \theta \kappa] \cup \dot{\cup} r-u)$, we see that $\Sigma(w)=\sigma+\theta \kappa 2, \Sigma\left(w^{\prime}\right)=\sigma+\theta \kappa 3$. This contradicts the commutativity of $s$, and so $s[>\gamma]$ must be finite for some $\gamma<\theta \kappa$ : in particular, it follows this that $t$ is finite.

Let $x$ be any permutation of $s[\geqslant \theta \kappa]$, and put $y=x-\theta \kappa$; of course $y$ is the corresponding permutation of $t$. Then $r \dot{\cup} x \stackrel{\bullet}{\bullet}[\leqslant \theta]$ is a permutation of $s$, and from arguments used in the preceding section we conclude that $\Sigma(r \dot{\cup} x \dot{\cup} s[\leqslant \theta])=\Sigma(r \dot{\cup} y \dot{\cup} s[\leqslant \theta])$; moreover, if $y, y^{\prime} \in P(t)$ are such that $\Sigma(y) \neq \Sigma\left(y^{\prime}\right)$, then $\Sigma\left(r\right.$ ن y $\left.\cup^{\circ} s[\leqslant \theta]\right) \neq \Sigma\left(r\right.$ ن $\left.y^{\prime} \cup \dot{\cup} s[\leqslant \theta]\right)$. Hence $t$ must be commutative.

We have thus shown that the three conditions are necessary. Assume now that they hold, and put $\psi=\sup \left\{s_{\xi} ; s_{\xi}<\theta \kappa\right\}$. Since $s[>\gamma]$ is finite for some $\gamma<\theta \kappa$, we must have $\psi<\theta \kappa$, and we claim that $s[\leqslant \psi]$ is commutative with $\Sigma(s[\leqslant \psi])=\theta \kappa$.

We are given that $s[\leqslant \theta]$ is commutative, and can proceed as before to show that $\Sigma(s[\leqslant \theta])=\theta \kappa$. Let $r$ be any permutation of $s[\leqslant \psi]$, and put $u=r[\leqslant \theta]$. By definition of $\theta$ we have $o(r-u)<\kappa$, and so $\Sigma(r-u)<\psi \kappa$. But $\psi=\theta \delta+\mu$ for some $\delta<\kappa$ and some $\mu<\theta$, whence $\psi<\theta(\delta+1)$. Since $\kappa$ is initial, we have $\psi \kappa \leqslant \theta(\delta+1) \kappa=\theta \kappa$, and so $\Sigma(r-u)<\theta \kappa=$ $\Sigma(u)$, the last equality following from $u \in P(s[\leqslant \theta])$. As $u$ is cofinal in $r$ Lemma 3 tells us that $\Sigma(r)=\theta \kappa$.

Let $w$ be any permutation of $s$, and put $v=w[\geqslant \theta \kappa]$. Then we know that $v$ is finite and therefore not cofinal in $w$. We have just seen that $\Sigma(w-v)=$ $\theta \kappa$, and so the results obtained in the previous sections show that $\Sigma(w)=$ 
$\Sigma(v)+\theta \kappa=\Sigma(v \circ \theta \kappa)+\theta \kappa$. But of course $v \circ \theta \kappa$ is a permutation of $t$.

It follows immediately that $s$ is commutative.

4. We turn our attention now to the problem of determining commutativity criteria for $\mu$-sequences, where $\mu$ is an infinite, noninitial prime component. We recall that in the preceding section the invariant $M(s)$ was defined to be $\min \{\gamma ;|o(s[>\gamma])|<|o(s)|\}$; in this section we use the related parameter $m(s)=\min \{\gamma ;|o(s[<\gamma])|=|o(s)|\}$. Obviously $m(s) \leqslant M(s)$.

An ordinal $\tau \geqslant \omega$ is sometimes called a " $\delta$-number" if $\beta \tau=\tau$ for every ordinal $\beta$ with $0<\beta<\tau$. It is not difficult to show that if $\alpha$ is any infinite ordinal, then there exists a maximum $\delta$-number $\tau$ with $\tau \leqslant \alpha$; we denote this $\tau$ by " $D(\alpha)$ ".

Let $\rho$ be an infinite prime component; the initial ordinal $\kappa$ defined by $|\kappa|=|\rho|$ will be denoted by " $K(\rho)$ ", and the ordinal $\gamma$ defined by $\rho=K(\rho) \gamma$ will be denoted by " $Q(\rho)$ ". We commence this section with the following:

THEOREM 12. Let $s$ be a $\rho$-sequence where $\rho$ is an infinite prime component such that $\rho=Q(\rho)$. Put $\kappa=K(\rho), \delta=D(\rho), \theta=m(s)$, and $r=s[\geqslant \theta \rho]$. For each $\beta \leqslant \theta \rho$, let $\mu_{\beta}$ be the least ordinal $\psi$ such that $\theta \rho \leqslant \beta \psi$. Then $s$ is commutative if and only if the following hold:

(1) $r \circ \theta \rho$ is finite and commutative;

(2) for each $\beta$ with $\theta \delta \leqslant \beta \leqslant \theta \rho$, there exists $\gamma<\beta$ such that $|o(s[\geqslant \gamma,<\beta])|<\left|\mu_{\beta}\right|$.

Proof. Assume firstly that the conditions hold. Then we must have $o(s-r)=\rho$, and we claim that $s-r$ is commutative with $\Sigma(s-r)=\theta \rho$. For take any permutation $t$ of $s-r$. Since $\kappa \rho=\rho$, we can express $t$ as $\stackrel{\bullet}{\xi<\rho}^{\xi}$, where each $t^{\xi}$ is a segment of $t$ having length $\kappa$. But from the definition of $\theta$ it follows that $\theta \leqslant \sup \left\{t_{\xi}^{\xi} ; \zeta<\kappa\right\}$ for each $\xi<\rho$, and so $\Sigma\left(t^{\xi}\right) \geqslant \theta$. Thus $\Sigma(t) \geqslant \theta \rho$.

Therefore if $s-r$ is not commutative, there is a permutation $t$ of $s$ and a proper initial segment $t^{\prime}$ of $t$ such that $\Sigma\left(t^{\prime}\right) \geqslant \theta \rho$; we may assume that $t$ and $t^{\prime}$ have been selected in such a manner that the parameter $\beta=\sup \left\{t_{\xi}^{\prime}\right.$; $\left.\xi<o\left(t^{\prime}\right)\right\}$ has been minimized.

Clearly we have $t_{\xi}^{\prime}<\theta \rho$ for all $\xi<o\left(t^{\prime}\right)$, and so $\Sigma\left(t^{\prime}\right)=\theta \rho$. But $\Sigma\left(t^{\prime}\right) \leqslant$ $\beta o\left(t^{\prime}\right)<\beta \rho$, whence it follows that $\beta>\theta$. Thus $\beta=\theta \psi+\mu$ for some $\psi$ and some $\mu<\theta$, and either $\psi>1$ or $\mu>0$. Suppose that $\psi<\delta$; then $\beta \delta=(\theta \psi$ $+\mu) \delta=\theta \psi \delta=\theta \delta$, and so $\beta \rho=\theta \rho$. But this yields $\Sigma\left(t^{\prime}\right)=\beta \rho>\beta o\left(t^{\prime}\right)$, a contradiction. Hence $\beta \geqslant \theta \delta$.

Applying (2) to $\beta$, we obtain $\gamma<\beta$ such that $|o(s[\geqslant \gamma,<\beta])|<\left|\mu_{\beta}\right|$; put $u=t^{\prime}[\geqslant \gamma,<\beta]$. Then $\Sigma(u)<\beta \mu_{\beta}$, and so $\Sigma(u)<\theta \rho$, since it is easily verified that $\beta \mu_{\beta}=\theta \rho$. Thus Result 4 implies that $\Sigma\left(t^{\prime}-\mu\right) \geqslant \theta \rho$, and since the reverse inequality is obvious, we arrive at $\Sigma\left(t^{\prime}-u\right)=\theta \rho$. Now put 
$v=t^{\prime}-u \stackrel{0}{u} \cup^{0} t-t^{\prime}$; then $v$ is a permutation of $s-r$ with $t^{\prime}-u$ as a proper initial segment. However, we have just seen that $\Sigma\left(t^{\prime}-u\right)=\theta \rho$, and it is clear that $\sup \left\{\left(t^{\prime}-u\right)_{\xi} ; \xi<o\left(t^{\prime}-u\right)\right\} \leqslant \gamma<\beta$, contradicting our choice of $\beta$. Thus $s-r$ is commutative, from which we deduce that $\Sigma(s-r)$ $=\theta \rho$. But now condition (1), combined with results from the preceding sections, tells us that $s$ is commutative.

We must now show that the conditions are necessary. To this end we put $t=s[<\theta]$ and observe that by definition of $\theta$ there is an arrangement $t^{0}$ of $t$ such that $o\left(t^{0}\right)=\rho$. Then $\Sigma\left(t^{0}\right)=\theta \rho$.

Obviously $\Sigma\left(t^{0}\right) \leqslant \theta \rho$, and as before we can express $t^{0}$ as $\cup_{\xi<\rho} t^{\alpha^{\xi}}$ with $o\left(t^{0^{\xi}}\right)=\kappa$ for each $\xi<\rho$, and note that $\Sigma\left(t^{0^{f}}\right) \geqslant \theta$ for each such $\xi$. Thus $\Sigma\left(t^{0}\right) \geqslant \theta \rho$.

Let $u$ be any arrangement of $s-t$ with $o(u)<\rho$, and put $v=u \cup^{0} t^{0}$. Then $v \in P(s)$ and $\Sigma(v)=\alpha+\theta \rho$ for some $\alpha$. Take any $r^{0} \in P^{a}(r)$ such that $r^{0}$ is a subsequence of $u$, and take any $x \in P^{a}(r)$ with $o(x)<\rho$; then $u-r^{0} \stackrel{0}{x} \dot{\cup} t^{0}$ is a permutation of $s$.

Suppose that (1) fails: then from the proof of Theorem 3 it follows that $\Sigma(x \circ \theta \rho) \neq \Sigma(y \circ \theta \rho)$ for some arrangements $x, y$ of $r$ with $o(x)=o(y)+$ $1<\rho$. But then $\Sigma(x)+\theta \rho \neq \Sigma(y)+\theta \rho$, and it now follows from our previous observation that $s$ is not commutative.

Now suppose that (2) fails; then there exists $\beta$ with $\theta \delta \leqslant \beta \leqslant \theta \rho$ such that $|o(s[\geqslant \gamma,<\beta])| \geqslant\left|\mu_{\beta}\right|$ for every $\gamma<\beta$. An exercise in manual labour now shows that there exists a subsequence $u$ of $s[\geqslant \theta,<\beta]$ such that for some arrangement $v$ of $u$ with $o(v)<\rho$ we have $\Sigma(v)=\theta \rho$. If $\beta$ is not a prime component, then this is obvious, for we must have $\psi \mu_{\beta}=\theta \rho$ for some $\psi<\beta$. If $\beta$ is a prime component, then this last statement is not necessarily true, but in this case we can always find an arrangement $v$ of some subsequence $u$ of $s[\geqslant \theta,<\beta]$ such that $v$ has the form $\cup_{\zeta<\mu_{\beta}} v^{\zeta}$, where the $v^{\zeta}$ are increasing sequences having common limit $\beta$ and common length $\tau$, with $\tau \mu_{\beta}<\rho$. The details are straightforward, but rather long and tedious.

Having obtained one such sequence $v$, we can now obtain $n$ such sequences for any given positive integer $n$, simply by splitting $v$ into appropriate subsequences. We have already seen, however, that there is an arrangement $t^{0}$ of $s[<\theta]$ such that $o\left(t^{0}\right)=\rho$ and $\Sigma\left(t^{0}\right)=\theta \rho$. It follows that for each positive integer $n$ there is a permutation $s^{n}$ of $s$ such that $\Sigma\left(s^{n}\right)=\alpha_{n}+\theta \rho(n+1)$ for some ordinal $\alpha_{n}$. It is clear from this, however, that $s$ cannot be commutative.

We must now deal with the case in which (in the notation of the above theorem) $\rho \neq Q(\rho)$. This occurs if and only if $\rho=\kappa^{n} \nu$ for some positive integer $n$ and some prime component $\nu<\kappa$, where either $n>1$ or $\nu>1$. We distinguish two possibilities, $\operatorname{cf}(\theta)=\kappa$ and $\operatorname{cf}(\theta)<\kappa$; obviously $\operatorname{cf}(\theta)>\kappa$ is impossible. 
THEOREM 13. Let $s$ be a $\rho$-sequence, with $\rho$ an infinite, noninitial prime component, and put $\kappa=K(\rho), \tau=Q(\rho), \delta=D(\rho), \theta=m(s)$, and $r=$ $s[\geqslant \theta \tau]$. For each $\beta \leqslant \theta \tau$, let $\nu_{\beta}$ be the least ordinal $\psi$ such that $\beta \psi \geqslant \theta \tau$. Assume that $\rho \neq \tau$ and that $\operatorname{cf}(\theta)=\kappa$.

Then $s$ is commutative if and only if the following hold:

(1) $r \circ \theta \tau$ is finite and commutative;

(2) if $\tau<\delta$, then for each $\beta$ with $\theta \leqslant \beta \leqslant \theta \tau$, there exists $\gamma<\beta$ such that $|o(s[\geqslant \gamma,<\beta])|<\left|\nu_{\beta}\right|$;

(3) if $\delta \leqslant \tau$, then for all $\beta$ with $\theta \delta \leqslant \beta \leqslant \theta \tau$, there exists $\gamma<\beta$ such that $|o(s[\geqslant \gamma,<\beta])|<\left|\nu_{\beta}\right|$.

Proof. Very similar to that of Theorem 12. We wish to show that $s-r$ is commutative, with sum $\theta \tau$. Take any $t \in P(s-r)$; then we can express $t$ as $\dot{\cup}_{\zeta<\tau} t^{\zeta}$ with $o\left(t^{\zeta}\right)=\kappa$ for each $\zeta<\tau$, and conclude via an argument similar to that used previously, that $\Sigma(t) \geqslant \theta \tau$.

Thus if $s-r$ is not commutative, then there exists some proper initial segment $u^{\prime}$ of some $u \in P(s-r)$ with $\Sigma\left(u^{\prime}\right) \geqslant \theta \tau$, and we may assume that $u$ and $u^{\prime}$ have been chosen in such a way that $\beta=\sup \left\{u_{\xi}^{\prime} ; \xi<o\left(u^{\prime}\right)\right\}$ is minimized. Now if $\beta<\theta$, then we can obtain a contradiction in the following manner. We can express $u^{\prime}$ as $\dot{\cup}_{\zeta<\psi} v^{\zeta} \cup v^{\prime}$, where $\psi<\tau$ is some ordinal, $o\left(v^{\zeta}\right)=\kappa$ for each $\zeta<\psi$, and $o\left(v^{\prime}\right)<\kappa$. From $\beta<\theta$ we deduce that $\Sigma(x)=\theta$ for some $x$, with $x$ either an initial segment of $v^{\prime}$ or a proper initial segment of some $v^{\zeta}$. But in either case we arrive at $\operatorname{cf}(\theta) \leqslant o(x)<\kappa$, a contradiction. Furthermore, if $\delta \leqslant \tau$, then we can argue as in the proof of Theorem 12 to obtain $\beta \geqslant \theta \delta$. The remainder of this proof parallels the previous one.

Clearly Theorems 12 and 13 could be fused; we feel, however, that the presentation given here highlights more clearly the essential aspects of the proof.

THEOREM 14. Let $s$ be a $\rho$-sequence, where $\rho$ is an infinite, noninitial prime component, and put $\kappa=K(\rho), \delta=D(\rho), \theta=m(s)$, and $r=s[\geqslant \theta \rho]$. Assume that $\rho \neq Q(\rho)$ and that $\operatorname{cf}(\theta)<\kappa$. For each $\beta \leqslant \theta \rho$, let $\mu_{\beta}$ be as in Theorem 12. Then $s$ is commutative if and only if

(1) $r \circ \theta \rho$ is finite and commutative;

(2) for each $\beta$ with $\theta \delta \leqslant \beta \leqslant \theta \rho$, there exists $\gamma<\beta$ such that $|o(s[\geqslant \gamma,<\beta])|<\left|\mu_{\beta}\right|$

(3) $\theta=\kappa^{\alpha}$ for $N O \alpha>0$.

Proof. Assume that the conditions hold, and let $t$ be any permutation of $s[\leqslant \theta]$. Clearly we can express $t$ as $\cup_{\zeta<Q(\rho)} t^{\zeta}$ with $o\left(t^{\zeta}\right)=\kappa$ and $m\left(t^{\zeta}\right)=\theta$ $=M\left(t^{\zeta}\right)$ for each $\zeta<Q(\rho)$. Now $\operatorname{cf}(\kappa)=\operatorname{cf}(\theta)<\kappa$, and so $\kappa$ is singular. Each $t^{\zeta}$ is bounded in the sense of the previous section, and condition (3) 
above is the amalgamation of conditions (1), (2) of Theorem 10. Thus $\Sigma\left(t^{\zeta}\right)=\theta \kappa$ for each $\zeta<Q(\rho)$, and so $\Sigma(t)=\theta \rho$. Therefore $s[\leqslant \theta]$ is commutative and $\Sigma(s[\leqslant \theta])=\theta \rho$.

Suppose that $s-r$ is not commutative; if follows from the preceding paragraph that there is a permutation $u$ of $s-r$ having a proper initial segment $u^{\prime}$ with $\Sigma\left(u^{\prime}\right)=\theta \rho$, and we may assume that $u, u^{\prime}$ have been chosen so that $\beta=\sup \left\{u_{\xi}^{\prime} ; \xi<o\left(u^{\prime}\right)\right\}$ has been minimized. The remainder of the proof of the sufficiency of our conditions now goes through exactly as in the proof of Theorem 12.

Suppose that condition (3) fails. There certainly exist arrangements $t, u$ of $s[\geqslant \theta]$ and $s[<\theta]$ respectively such that $o(t)<o(u)=\rho$. Express $u$ as $\bigcup_{\zeta<Q(\rho)} u^{\zeta}$ with $o\left(u^{\zeta}\right)=\kappa$ for $\zeta<Q(\rho)$; from Theorem 9 we know that there exists a permutation $x^{\zeta}$ of $u^{\zeta}$ with $\Sigma\left(x^{\zeta}\right)=\theta \kappa$, and from the proof of Theorem 10 (the case $\eta=\lambda$ ) we know that there exists a permutation $y^{\zeta}$ of $u^{\zeta}$ with $\Sigma\left(y^{5}\right)=\theta$. Thus there exist permutations $x, y$ of $u$ such that $\Sigma(x)=\theta \rho$ and $\Sigma(y)=\theta Q(\rho)$. Hence $s$ is not commutative.

On the other hand, if condition (3) holds but either (1) or (2) fails, then we can follow the proof of Theorem 12 to show that $s$ is not commutative.

5. Thus far in this paper we have dealt with commutativity criteria for $\alpha$-sequences where $\alpha$ is an infinite prime component. We now turn to the case in which $\alpha$ is an infinite successor ordinal. Let $s$ be any nonempty sequence: we define $L(s)$ to be $\min \left\{s_{\xi} ; \xi<o(s)\right\}$ and $\Gamma(s)$ to be $\left\{\xi<o(s) ; s_{\xi} \neq L(s)\right\}$. Obviously $L(s)$ is an invariant of $s$ and $\Gamma(s)$ is a quasi-invariant in the sense that if $f: o(s) \rightarrow o(s)$ is any bijection and $t \in P(s)$ is defined by $t_{\xi}=s_{f(\xi)}$, $\xi<o(s)$, then $\Gamma(t)=f^{-1} \Gamma(s)$.

THEOREM 15. Let $s$ be a commutative $\gamma+1$-sequence, with $\gamma$ an infinite ordinal. Then $\Gamma(s)$ is finite, and there is an ordinal $\delta \geqslant L(s) \omega$ such that for each $\xi \in \Gamma(s)$ we have $s_{\xi}=(\delta+L(s)) m_{\xi}$ for some positive integer $m_{\xi}$.

Proof. If $\Gamma(s)$ is empty, then the result holds trivially; hence we may assume that this is not the case. Let $r$ be a permutation of $s$ such that $r_{0}=L(s)<r_{\gamma}$, and put $\alpha=\Sigma\left(\left(r_{\zeta}\right)_{0<\zeta<\gamma}\right)$. Then $\Sigma(r)=r_{0}+\alpha+r_{\gamma}$, and since $s$ is commutative we obtain $r_{0}+\alpha+r_{\gamma}=r_{\gamma}+\alpha+r_{0}$. But because $r_{0}=L(s)$ and $\gamma$ is infinite we must have $r_{0}+\alpha=\alpha$. Thus if we also had $r_{\gamma}+\alpha=\alpha$, then we could obtain $\alpha+r_{\gamma}=\alpha+r_{0}$, and hence $r_{\gamma}=r_{0}$, contrary to assumption. Therefore $\alpha<r_{\gamma} k$ for some number $k$, from which it follows that $r_{0}+r_{\gamma}=r_{\gamma}$. But this gives $r_{\gamma}+\left(\alpha+r_{0}\right)=r_{0}+\alpha+r_{\gamma}=\alpha+$ $r_{\gamma}=\left(\alpha+r_{0}\right)+r_{\gamma}$, and so Result 1 tells us that there is an ordinal $\sigma$ and numbers $p, q$ such that $\alpha+r_{0}=\sigma p$ and $r_{\gamma}=\sigma q$. Let $\theta$ be the least such $\sigma$; expressing $\theta$ as $\tau+\mu$ with $\tau$ being the maximum prime component not 
exceeding $\theta$, we see that $\mu<\tau$, and we have $r_{\gamma}=\tau q^{\prime}+\mu$ for some number $q^{\prime} \neq 0$.

Suppose that $r_{\psi} \neq L(s)$ for some $\psi<\gamma$. Then $r_{\psi} \leqslant \alpha \leqslant r_{\gamma} k$, and by repeating the above argument with the permutation $r^{0}=\left(r_{\xi}\right)_{0 \leqslant \zeta<\psi} \stackrel{\cup}{\cup}\left(r_{\gamma}\right) \dot{\ominus}$ $\left(r_{\xi}\right)_{\psi<\zeta<\gamma} \dot{\cup}\left(r_{\psi}\right)$ of $s$, we see that $r_{\gamma} \leqslant r_{\psi} k^{\prime}$ for some number $k^{\prime}$. It follows that $r_{\psi}=\tau n+\nu$ for some number $n \neq 0$ and some $\nu<\tau$. Now $\Sigma(r)=\alpha+$ $\tau q^{\prime}+\mu$ and $\Sigma\left(r^{0}\right)=\alpha^{0}+\tau n+\nu$ for some $\alpha^{0}$. But $\Sigma(r)=\Sigma\left(r^{0}\right), \mu, \nu<\tau$, and $\tau$ is a prime component. Thus we must have $\mu=\nu$. We have therefore shown that there is an ordinal, viz. $\theta$, for which we have, for each $\xi \in \Gamma(s)$, a number $m_{\xi}>0$ such that $s_{\xi}=\theta m_{\xi}$.

Suppose that $\Gamma(s)$ is infinite and put $X=\Gamma(s)-\{\gamma\}$. Then of course $X$ is infinite, and so if we choose $\chi \in X$ such that $r_{x}=\min \left\{r_{\xi} ; \xi \in X\right\}$, then

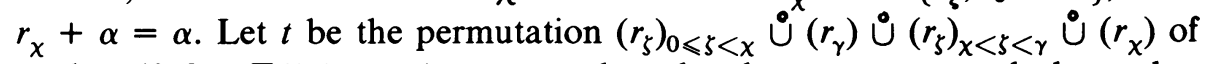
$s$; then if $\beta=\Sigma\left(\left(t_{\zeta}\right)_{0<\zeta<\gamma}\right)$, we can show by the argument used above that $\beta \leqslant r_{\chi} c$ for some number $c$. However, $r_{\gamma}=\theta n$ and $r_{\chi}=\theta n^{\prime}$ for some numbers $n, n^{\prime}>0$, and so $\theta n^{\prime} \leqslant \alpha \leqslant \theta n k$ and $\theta n \leqslant \beta \leqslant \theta n^{\prime} c$, from which it follows that $\alpha \leqslant \beta p$ for some number $p$. Thus $\beta+\alpha>\alpha$. But $\beta \leqslant r_{x} c$ and $r_{\chi}+\alpha=\alpha$, whence $\beta+\alpha=\alpha$. This contradiction shows that $\Gamma(s)$ must be finite.

It remains to show that $\theta=\delta+L(s)$ for some $\delta \geqslant L(s) \omega$. We have already shown that $L(s)+\alpha=\alpha$, whence $\alpha \geqslant L(s) \omega$, and that $\alpha+L(s)=$ $\theta p^{\prime}$ for some number $p^{\prime}$, where $\alpha=\Sigma\left(\left(r_{\zeta}\right)_{0<\zeta<\gamma}\right)$. Therefore $\theta p^{\prime} \geqslant L(s) \omega$, and so $\theta \geqslant L(s) \omega$. Furthermore, from $\theta p^{\prime}=\alpha+L(s)$ we see that $L(s)$ is a remainder of $\theta$, i.e., $\theta=\delta+L(s)$ for some $\delta$. If $\delta<L(s) \omega$, then of course $\theta<L(s) \omega$, contradicting the above inequality. Hence $\delta \geqslant L(s) \omega$.

The problem of determining commutativity criteria for a sequence whose length is an infinite successor ordinal splits into two cases.

THEOREM 16. Let $s$ be a $\rho k+1$-sequence, where $\rho$ is an infinite prime component and $k$ is a positive number.

Then $s$ is commutative if and only if $\Gamma(s)$ is finite and for each $\xi \in \Gamma(s)$ there is a number $m_{\xi}>0$ such that $s_{\xi}=L(s)(\rho+1) m_{\xi}$.

Proof. Assume that $s$ is commutative; then Theorem 15 tells us that $\Gamma(s)$ is finite and that there is an ordinal $\delta \geqslant L(s) \omega$ such that for each $\xi \in \Gamma(s)$ we have $s_{\xi}=(\delta+L(s)) m_{\xi}$ for some number $m_{\xi}>0$. We show firstly that $\delta=\theta+L(s) \rho$ for some ordinal $\theta$. We may of course assume that $\Gamma(s) \neq \varnothing$.

Take any $\zeta \in \Gamma(s)$; then $s_{\zeta}=\delta m_{\zeta}+L(s)$. By repeating the relevant arguments used in the proof of Theorem 15 we can show that $\Sigma(s) \leqslant s_{\zeta} n$ for some $n$. But of course $L(s) \rho \leqslant \Sigma(s)$, and so we have $L(s) \rho \leqslant \delta m_{\zeta} n+L(s)$. Since $\rho$ is a prime component and $\rho>1, L(s) \rho$ is also a prime component, and it follows that $\delta \geqslant L(s) \rho$. 
Let $r$ be some permutation of $s$ for which $r_{\rho k}>L(s)$ : then we have $r_{\rho k}=\delta m+L(s)$ for some number $m>0$, and so $\Sigma(r)=\eta+\delta+L(s)$ for some ordinal $\eta$. But $\Gamma(s)$ is finite, and so by taking a permutation $t$ of $s$ for which $t_{\xi}=L(s)$ for all $\xi \geqslant|\Gamma(s)|$, we see that the commutativity of $s$ implies $\Sigma(r)=\Sigma(t)=\lambda+L(s)(\rho+1)$ for some $\lambda$. Thus we have $\eta+\delta+L(s)=\lambda$ $+L(s)(\rho+1)$ and $\delta \geqslant L(s) \rho$, and from these we conclude that $\delta=\theta+$ $L(s) \rho$ for some $\theta$.

We have thus far shown the existence of an ordinal $\theta$ such that $s_{\zeta}=(\theta+$ $L(s)(\rho+1)) m_{\zeta}$ for each $\zeta \in \Gamma(s)$. Suppose that $\theta<L(s) \rho \omega$, i.e., $\theta=L(s) \rho n$ $+\mu$ for some number $n$ and some $\mu<L(s) \rho$. Since $L(s) \rho$ is a prime component, it follows that, for each $\zeta \in \Gamma(s), s_{\zeta}=L(s)(\rho(n+1)+1) m_{\zeta},=$ $L(s)(\rho+1) k$, where $k=m_{\zeta}$ if $n=0$ and $k=n m_{\zeta}$ otherwise. This is the required form for $s_{\zeta}$, and so the necessity of our conditions will have been proved if we can show that $\theta \geqslant L(s) \rho \omega$ leads to a contradiction.

Assume that $\theta \geqslant L(s) \rho \omega$ and consider the permutation $r$ of $s$ used above. We have $\Sigma(r)=\eta^{\prime}+L(s)(\rho n+1)$ where $n$ is some positive number and $\eta^{\prime}$ is some ordinal with $R\left(\eta^{\prime}\right) \geqslant L(s) \rho \omega$. Now $\Gamma(s)$ is finite, and so there is a permutation $u$ of $s$ such that for some $\zeta^{0}<\rho k$ we have $u_{\zeta^{0}}=r_{\rho k}$ and $u_{\xi}=L(s)$ for every $\xi$ with $\zeta^{0}<\xi \leqslant \rho k$. Express $\zeta^{0}$ as $\rho c+\mu$ with $c<k$ and $\mu<\rho$ : then for some $\lambda$ we have $\Sigma(u)=\lambda+\sigma^{\prime}+L(s)(\rho(n+k-c)+1)$, where the ordinal $\sigma^{\prime}$ is such that $r_{\rho k}=\sigma^{\prime}+L(s)(\rho n+1)$. From the expression for $\Sigma(r)$ we see that $R\left(\sigma^{\prime}\right) \geqslant L(s) \rho \omega$, and by the commutativity of $s$ we have $\Sigma(r)=\Sigma(u)$. This however leads to the contradiction $\rho(n+k-c)$ $+1=\rho n+1$. Thus our conditions are necessary.

Suppose now that the conditions hold, and let $t$ be a permutation of $s$. Put $r=t[>L(t)]$; by invariance we have $|o(r)|=|o(s[>L(s)])|$, and so $o(r)=q$ for some number $q$. For each $j \leqslant q$ let $t^{j}$ be that segment of $t$ for which $t=\dot{\cup}\left\{t^{j} \stackrel{\bullet}{\cup}\left(r_{j}\right) ; j<q\right\} \stackrel{\bullet}{t^{q}}$, and for each such $j$ put $o\left(t^{j}\right)=\rho k_{j}+\tau_{j}$ for some number $k_{j}$ and some ordinal $\tau_{j}<\rho$. Then $\Sigma\left(t^{j} \dot{U}\left(r_{j}\right)\right)=L(s)\left(\rho\left(k_{j}+\right.\right.$ $\left.\left.m_{j}\right)+1\right)$ for each $j<q$, where $m_{j}$ is the number such that $r_{j}=L(s)(\rho+1) m_{j}$.

Since $o(t)=\rho k+1$, we must have $\tau_{q} \leqslant 1$ : it follows that $\Sigma(t)=L(s)(\rho n$ $+1)$, where $n=\Sigma\left\{k_{j}+m_{j} ; j<q\right\}+k_{q}$. But of course $\Sigma_{j<q} k_{j}=k$ and $\Sigma_{j<q} m_{j}=\Sigma\left\{m_{\xi} ; \xi \in \Gamma(s)\right\}$. Thus $n$ is independent of $t$, and so $s$ is commutative.

THEOREM 17. Let s be a $\gamma$-sequence, where $\gamma$ is an infinite successor ordinal that is not of the form $\rho k+1$ for any prime component $\rho$ and number $k$. Then $s$ is commutative if and only if $\Gamma(s)=\varnothing$.

Proof. The condition being obviously sufficient, we concentrate upon the necessity, and assume that $s$ is commutative and that $\Gamma(s) \neq \varnothing$. The condition upon $\gamma$ ensures that we can represent it in the form $\tau+\rho+1$, where $\rho$ is a 
prime component and $\tau$ is an ordinal with $\tau \geqslant \rho \omega$. From Theorem 15 we know that $\Gamma(s)$ is finite and that there is an ordinal $\delta$ such that for each $\xi \in \Gamma(s)$ we have $s_{\xi}=(\delta+L(s)) m_{\xi}$ for some number $m_{\xi}>0$. Furthermore, as in the proof of Theorem 16 we can show that $\delta=\theta+L(s) \rho$ for some $\theta$. Let $\psi$ be such that $\gamma=\psi+1$.

Let $t$ be a permutation of $s$ with $t_{\psi} \neq L(s)$, and let $m$ be the largest number $k$ such that $t_{\psi}=\mu+L(s)(\rho k+1)$ for some $\mu$. Thus either $\mu<L(s) \rho$ (in which case $\left.t_{\psi}=L(s)(\rho m+1)\right)$, or else $R(\mu) \geqslant L(s) \rho \omega$. But we can show as in the proof of Theorem 15 that $L(s) \tau \leqslant t_{\psi} p$ for some $p$, and so it must be the case that $t_{\psi}>L(s)(\rho m+1)$. Thus $R(\mu) \geqslant L(s) \rho \omega$, and it follows that $\Sigma(t)$ $=\nu+L(s)(\rho m+1)$ for some $\nu$ with $R(\nu) \geqslant L(s) \rho \omega$.

Since $\Gamma(s)$ is finite, there is a permutation $u$ of $s$ such that $u_{\tau}=t_{\psi}$ and $u_{\xi}=L(s)$ for each $\xi$ with $\tau<\xi<\gamma$. Then for some $\lambda$ with $R(\lambda) \geqslant L(s) \rho \omega$, we have $\Sigma(u)=\lambda+L(s)(\rho m+1)+L(s)(\rho+1)=\lambda+L(s)(\rho(m+1)+$ 1). Since $s$ is commutative, this yields the contradiction $\rho m+1=\rho(m+1)$ +1 .

6. We come to the final case, that in which $o(s)$ is an infinite limit ordinal but not a prime component. Since in this case $o(s)$ can be expressed as $\rho(\gamma+1)$, where $\rho$ is an infinite prime component and $\gamma$ is a nonzero ordinal, it is perhaps natural to expect that this case can be dealt with by amalgamating in some manner the results of $\S 4$ with either Result 2 or the results of $\S 5$, according as $\gamma$ is finite or infinite. Commutativity criteria can indeed be derived in this way, and this method of derivation sheds some interesting sidelights on ordinal series: for the purposes of this paper however, the following simpler method seems preferable.

Let $s$ be any sequence and $\alpha$ any ordinal. We define $P(s, \alpha)$ to be the set of all $\alpha$-sequences $t$ for which there exists an injection $f: \alpha \rightarrow o(s)$ such that $t_{\xi}=s_{f(\xi)}$ for each $\xi<\alpha$ : we put $S(s, \alpha)=\{\Sigma(t) ; t \in P(s, \alpha)\}$.

The invariant relevant to this section is $Q(s)=\min S(s, \rho)$, where we are taking $o(s)$ as $\rho(\gamma+1)$, with $\rho$ an infinite prime component and $\gamma$ a nonzero ordinal. It is clear from our definitions that $Q(s)$ is indeed an invariant of $s$, but an explicit determination of $Q(s)$ would take us too far afield: for those interested in problems like this, we refer to the work done by Anderson in [1]. For our purposes, the following simple result suffices:

LEMMA 6. Let $s$ be a sequence such that $o(s)=\rho(\gamma+1)$ for some infinite prime component $\rho$ and some nonzero ordinal $\gamma$. Then $Q(s)$ is an infinite prime component.

Proof. Let $t \in P(s, \rho)$ be such that $\Sigma(t)=Q(s)$; then because $\rho$ is an infinite prime component $t$ has a final segment $t^{\prime}$ with $o\left(t^{\prime}\right)=\rho$ and $\Sigma\left(t^{\prime}\right)=$ $R(Q(s))$. But $t^{\prime} \in P(s, \rho)$. Hence $R(Q(s))=Q(s)$ and so $Q(s)$ is a prime 
component. That $Q(s)$ is infinite is obvious.

THEOREM 18. Let $s$ be $a \rho(\gamma+1)$-sequence, where $\rho$ is a singular prime component and $\gamma$ is a nonzero ordinal. Put $\tau=Q(s)$, let $\theta$ be the least ordinal for which $\theta \rho \geqslant \tau$, and for each $\beta$ with $\theta \leqslant \beta \leqslant \tau$, let $\chi_{\beta}$ be the least ordinal such that $\beta \chi_{\beta} \geqslant \tau$.

Then $s$ is commutative if and only if the following hold:

(1) $s[\geqslant \tau]$ is finite and $s[\geqslant \tau \omega] \circ \tau$ is commutative;

(2) for each $\beta$ with $\theta \leqslant \beta \leqslant \tau$, there exists $\psi<\beta$ such that $|o(s[>\psi,<\beta])|$ $<\left|x_{\beta}\right|$.

Proof. Suppose that $s$ is commutative, but that $s[\geqslant \tau]$ is infinite. If $|o(s[\geqslant \tau])|<|\rho|$, then it is clear that $v \cup^{\circ} t \stackrel{\bullet}{r}$ is a permutation of $s$, where $r \in P(s[<\tau], \rho)$ is such that $\Sigma(r)=\tau, v$ is some fixed arrangement of $s[>\tau]-r$ such that $o(v)=\rho \gamma$, and $t$ is any arrangement of $s[\geqslant \tau]$. Since $t$ is an arbitrary arrangement of $s[\geqslant \tau]$, it is clear that we have contradicted the commutativity of $s$. If $|o(s[\geqslant \tau])| \geqslant|\rho|$, then we can select some subsequence $s^{\prime}$ of $s[\geqslant \tau]$ with $\left|o\left(s^{\prime}\right)\right|<|\rho|$, and argue along lines similar to the above to arrive at a contradiction. Thus $s[\geqslant \tau]$ must be finite. If $s[\geqslant \tau \omega]^{0} \bullet \tau$ is not commutative, then we can use the same argument, this time taking $v$ to be an arrangement of $s[<\tau \omega]-r$ with $o(v)=\rho \gamma$ and $t$ to be any permutation of $s[\geqslant \tau \omega]$. Thus if $s$ is commutative, then (1) must hold.

If condition (2) fails, then for some $\beta$ with $\theta \leqslant \beta \leqslant \tau$ we can select from some arrangement of $s[<\beta]$ a subsequence $t$ such that $\Sigma(t)=\tau$ and $o(t)=$ $\operatorname{cf}(\tau)=\operatorname{cf}(\rho)<\rho$. Then there is some $v \in P(s, \rho \gamma)$ and some $r \in P(s, \rho)$ such that $v \stackrel{\bullet}{t} \cup \stackrel{r}{r}$ is a permutation of $s$ and $\Sigma(r)=\tau$. By a "splitting" argument, such as has been used in previous sections, we can now show that $\Sigma(v)+\tau(n+1) \in S(s)$ for each $n>0$. Thus if $s$ is commutative, then (2) holds.

We have thus demonstrated the necessity of the conditions. Assume that they hold, and put $r=s[<\tau]$. Then clearly $s$ is commutative if $r$ is commutative. Let $t$ be any permutation of $r$, and express $t$ as $\cup_{\xi<\gamma} t^{\xi}$ with each $o\left(t^{\xi}\right)=\rho$. Naturally $\Sigma\left(t^{\xi}\right) \geqslant \tau$ for each $\xi \leqslant \gamma$, because $t^{\xi} \in P(s, \rho)$. On the other hand, we can proceed as in the proof of Theorem 12 to show that we cannot have $\Sigma\left(t^{\xi}\right)>\tau$ for any $\xi \leqslant \gamma$. Thus $\Sigma(t)=\tau(\gamma+1)$, and since $t$ was an arbitrary permutation of $r$, we have shown that $r$ is commutative.

THEOREM 19. Let $s$ be a $\rho(\gamma+1)$-sequence, where $\rho$ is a regular initial ordinal and $\gamma$ is a nonzero ordinal. Put $\tau=Q(s)$.

Then $s$ is commutative if and only if $s[\geqslant \tau]$ is finite and $s[\geqslant \tau \omega] \circ \tau$ is commutative.

PROOF. The necessity of our condition is proved as in the previous theorem. Assume it holds and put $r=s[<\tau]$; then $s$ is commutative if $r$ is. Take any 
permutation $t$ of $r$ and express $t$ as $\dot{\cup}_{\xi \leqslant \gamma} t^{\xi}$ with each $o\left(t^{\xi}\right)=\rho$. Then $\sum\left(t^{\xi}\right) \geqslant \tau$, whilst $\Sigma\left(t^{\xi}\right)>\tau$ yields the contradiction $\operatorname{cf}(\rho)=\operatorname{cf}(\tau)<o\left(t^{\xi}\right)=$ $\rho$. It follows as previously that $r$ is commutative.

The proofs of the two preceding theorems contain no arguments that have not previously appeared-in some guise or other-in this paper: we have therefore simply indicated their formats, rather than giving them in full.

\section{REFERENCES}

1. J. A. H. Anderson, The minimum sum of an arbitrary family of ordinals, J. London Math. Soc. (2) 7 (1973), 429-434.

2. S. Ginsburg, On the distinct sums of $\lambda$-type transfinite series obtained by permuting the elements of a fixed $\lambda$-type series, Fund. Math. 39 (1952), 131-132.

3. J. L. Hickman, A problem on series of ordinals, Fund. Math. 81 (1973), 49-55.

4. Concerning the number of sums obtainable from a countable series of ordinals by permutations that preserve the order-type, J. London Math. Soc. (2) 9 (1974), 239-244.

5. __ Some results on series of ordinals, Z. Math. Logik Grundlagen Math. 23 (1977), $1-18$.

6. W. Sierpiński, Sur les séries infinies de nombres ordinaux, Fund. Math. 36 (1949), 248-253.

7. , Cardinal and ordinal numbers, Warszawa, 1965.

Department of Mathematics, Instrtute of Advanced Studies, Australian National University, CANBerRa A.C.T. 2600, Australia 